\title{
Allocation of intensive care resources during an infectious disease outbreak: a rapid review to inform practice
}

Kirsten M. Fiest ${ }^{1,2}$, Karla D. Krewulak ${ }^{1}$ Kara M. Plotnikoff ${ }^{1}$, Laryssa G. Kemp ${ }^{1}$, Ken Kuljit S. Parhar ${ }^{1}$, Daniel J. Niven ${ }^{1,2}$, John B. Kortbeek ${ }^{1,3,4}$, Henry T. Stelfox ${ }^{1,2+}$ and Jeanna Parsons Leigh ${ }^{5,6^{*}+}$

\begin{abstract}
Background: The COVID-19 pandemic has placed sustained demand on health systems globally, and the capacity to provide critical care has been overwhelmed in some jurisdictions. It is unknown which triage criteria for allocation of resources perform best to inform health system decision-making. We sought to summarize and describe existing triage tools and ethical frameworks to aid healthcare decision-making during infectious disease outbreaks.

Methods: We conducted a rapid review of triage criteria and ethical frameworks for the allocation of critical care resources during epidemics and pandemics. We searched Medline, EMBASE, and SCOPUS from inception to November 3, 2020. Full-text screening and data abstraction were conducted independently and in duplicate by three reviewers. Articles were included if they were primary research, an adult critical care setting, and the framework described was related to an infectious disease outbreak. We summarized each triage tool and ethical guidelines or framework including their elements and operating characteristics using descriptive statistics. We assessed the quality of each article with applicable checklists tailored to each study design.

Results: From 11,539 unique citations, 697 full-text articles were reviewed and 83 articles were included. Fifty-nine described critical care triage protocols and 25 described ethical frameworks. Of these, four articles described both a protocol and ethical framework. Sixty articles described 52 unique triage criteria (29 algorithm-based, 23 pointbased). Few algorithmic- or point-based triage protocols were good predictors of mortality with AUCs ranging from 0.51 (PMEWS) to 0.85 (admitting SOFA > 11). Most published triage protocols included the substantive values of duty to provide care, equity, stewardship and trust, and the procedural value of reason.

Conclusions: This review summarizes available triage protocols and ethical guidelines to provide decision-makers with data to help select and tailor triage tools. Given the uncertainty about how the COVID-19 pandemic will progress and any future pandemics, jurisdictions should prepare by selecting and adapting a triage tool that works best for their circumstances.
\end{abstract}

Keywords: Triage, Critical care, Intensive care, Resource allocation, Medical ethics, Practice guideline, COVID-19

\footnotetext{
* Correspondence: J.ParsonsLeigh@dal.ca

${ }^{\dagger}$ Henry T. Stelfox and Jeanna Parsons Leigh are co-senior authors.

${ }^{5}$ Faculty of Health, School of Health Administration, Dalhousie University,

5850 College Street, Halifax, Nova Scotia B3H4R2, Canada

${ }^{6}$ Department of Critical Care Medicine, Faculty of Medicine, Dalhousie

University, 6299 South St, Halifax, Nova Scotia B3H4R2, Canada

Full list of author information is available at the end of the article
}

(c) The Author(s). 2020 Open Access This article is licensed under a Creative Commons Attribution 4.0 International License, which permits use, sharing, adaptation, distribution and reproduction in any medium or format, as long as you give appropriate credit to the original author(s) and the source, provide a link to the Creative Commons licence, and indicate if changes were made. The images or other third party material in this article are included in the article's Creative Commons licence, unless indicated otherwise in a credit line to the material. If material is not included in the article's Creative Commons licence and your intended use is not permitted by statutory regulation or exceeds the permitted use, you will need to obtain permission directly from the copyright holder. To view a copy of this licence, visit http://creativecommons.org/licenses/by/4.0/. The Creative Commons Public Domain Dedication waiver (http://creativecommons.org/publicdomain/zero/1.0/) applies to the data made available in this article, unless otherwise stated in a credit line to the data. 


\section{Background}

Five pandemics occurred in the last century: H1N1 (1918), H2N2 (1957-1958), H3N2 influenza (1968), the H1N1 virus (2009) [1-3], and the novel coronavirus SARS-CoV-2 (COVID-19) (2020) [4]. Viral infections caused by influenza or coronavirus may lead to organ failure, including respiratory illness, which can progress to hypoxemic respiratory failure and the acute respiratory distress syndrome, requiring admission to an intensive care unit (ICU) [5]. ICUs are specialized units wherein highly trained specialists work with a multidisciplinary team to deliver life sustaining therapies [6]. The availability of ICU beds varies across countries ranging from 0 (low-income countries such as South Sudan) to 59.5 (high-income countries such as Monaco) adult ICU beds per 100,000 population [7]. With the world's population over 7.8 billion [8], rapid viral spread during a pandemic can overwhelm ICU resources $[9,10]$ (e.g., staff, beds, ventilators, extracorporeal life support).

It is essential that hospitals have a strategy to ensure equitable and ethical resource allocation if the demand for ICU resources exceeds supply [11]. One such strategy is a triage protocol, which is a set of criteria that are enacted during resource scarcity to determine which patients should be admitted to an ICU or continue to receive care in an ICU. Recent reviews discuss the principle of triage and implementation of triage plans [12], quality of criteria to triage and transfer critically ill patients [13], and ethical principles used in disaster response $[14,15]$. However, it is unclear which triage criteria perform best. In light of the ongoing COVID-19 pandemic, we conducted a rapid review of the published triage literature to evaluate the validity of published triage protocols and the mortality prediction embedded within them to help inform health system-decisionmaking.

\section{Methods}

We conducted a rapid review to ensure timeliness of data in response to the COVID-19 pandemic and adhered to the Preferred Reporting Items for Systematic Reviews and Meta-Analyses standards where possible (as no guideline for rapid reviews exists) [16] (Supplementary Appendix 2, Additional File 1). The search strategy included the following: (1) terms for critical care (e.g., ICU, intensive care, mechanical ventilation); (2) terms for pandemics, epidemics, and infectious disease outbreaks (e.g., avian influenza, Severe Acute Respiratory Syndrome [SARS], Middle East Respiratory Syndrome [MERS], COVID-19); and (3) terms for triage criteria, resource allocation, and ethical frameworks (e.g., triage, resource allocation) (see Supplementary Appendix 2, Additional File 1). The search was executed on November 3, 2020, in three databases: MEDLINE (Ovid),
EMBASE (Ovid), and SCOPUS (Elsevier). References were exported and managed using EndNote X9 (Clarivate Analytics).

To ensure inter-rater agreement, a random sample of $10 \%$ of included articles were pilot tested independently by three reviewers (KK, KP, LK) with $92 \%$ agreement. The remaining titles and abstracts were screened independently by one of three reviewers (KK, KP, LK). Studies were included if they were published in the English language and were (1) primary research and other research, including consensus-based study designs; (2) targeted critically ill adults (i.e., patients with lifethreatening respiratory, cardiovascular, or neurological illness or other illnesses necessitating life sustaining therapies only provided in an ICU and are $\geq 18$ years old [or an adult as defined by the study]); and (3) described development or evaluation of triage criteria used on all patients (e.g., COVID and non-COVID) to allocate critical care resources during an infectious disease outbreak. Citations deemed eligible by any reviewer at the title and abstract stage were included for full-text review. Fulltext articles were similarly screened independently and in duplicate by three reviewers (KK, KP, LK). Disagreements were resolved by discussion or consensus with a fourth reviewer (KF). Reference lists were examined for any additional relevant studies not identified in the original search.

The following data were recorded on a standardized form in Excel: study information (e.g., country of study conduct, year published, study design), participant characteristics (e.g., study population, sample size), and outcomes (e.g., triage guideline creation, evaluation of triage criteria, and triage protocols). Data are reported using descriptive statistics.

The quality of included quantitative studies was assessed using the Joanna Briggs Institute Critical Appraisal tools for case control, cohort, cross-sectional, diagnostic test accuracy, quasi-experimental, qualitative, or text and opinion studies [17-22]. The quality of Delphi techniques was assessed based on proposed recommendations by Jünger and colleagues. The quality of guidelines was assessed using the 23-item AGREE Reporting Checklist. In all cases, a higher number indicated better methodological quality.

\section{Results}

The literature search identified 11,539 unique citations (Fig. 1). After assessing titles and abstracts for eligibility, 697 articles were included for full-text review. Eightythree articles were included in the final rapid review. The most common reasons for exclusion included not original research $(249 / 614,40.6 \%)$ or not reporting on the development or evaluation of triage criteria used on 


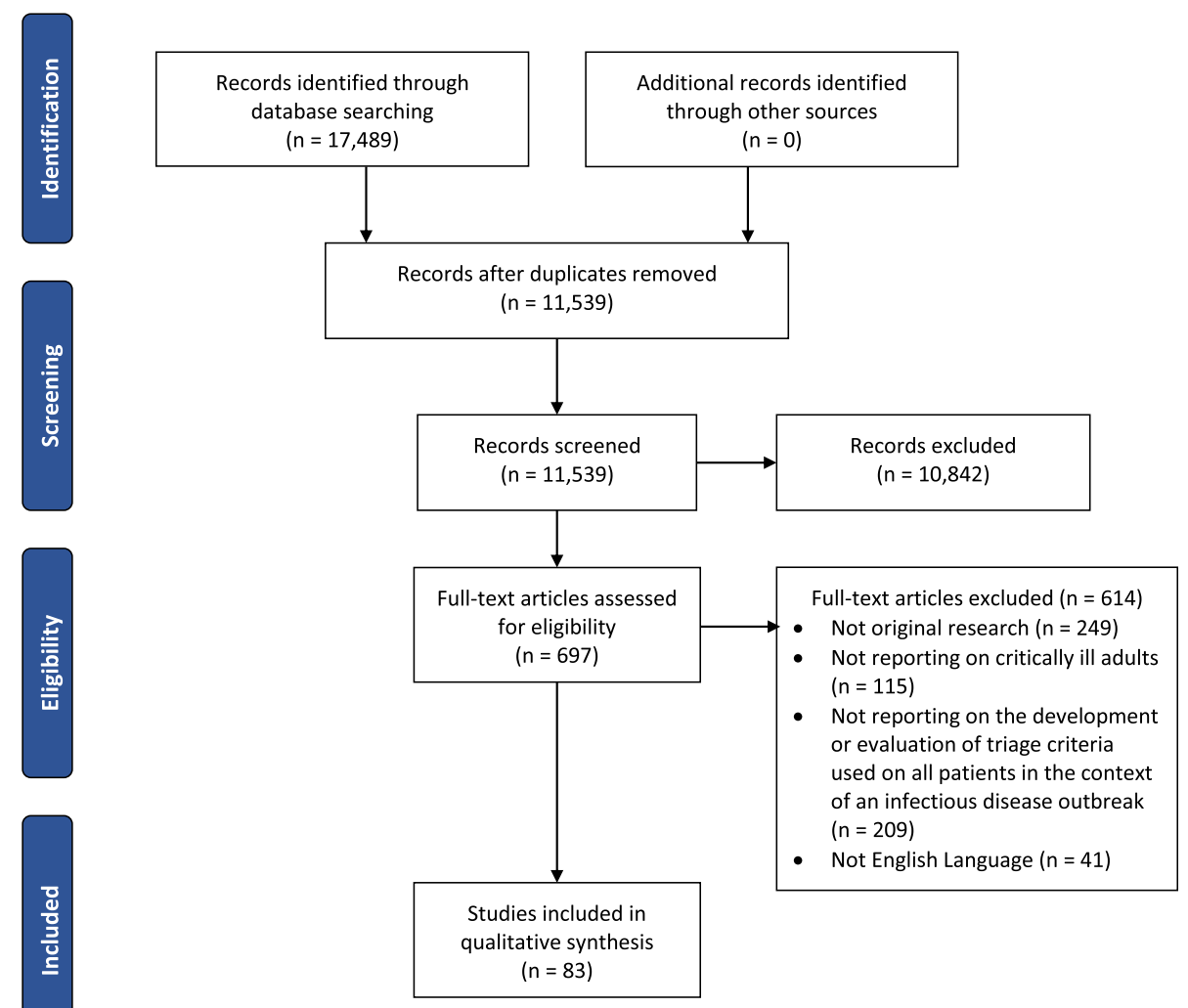

Fig. 1 Preferred Reporting Items for Systematic Reviews and Meta-Analyses (PRISMA) diagram of study selection

all patients in the context of an infectious disease outbreak (209/614, 34.0\%(Fig. 1).

Supplementary Table 1, Additional File 1 presents the characteristics of the 83 included articles, published from 2005 to 2020. Studies were conducted in North America (43/83, 51.8\%), Europe (24/83, 28.9\%), Oceania (6/83, 7.2\%), across multiple continents $(5 / 83,6.0 \%)$, Asia $(4 / 83,4.8 \%)$, and one was unclear $(1 / 83,1.2 \%)$. Observational studies included retrospective cohort (17/83, $20.5 \%)$, prospective cohort $(8 / 83,9.6 \%)$, cross-sectional (4/83, 4.8\%), mixed methods/computer modeling (each $2 / 83,2.4 \%$ ), and pre-post/qualitative (each 1/83, 1.2\%). Several studies were categorized as text and opinion (48/ $83,57.8 \%$ ), which was further defined as studies that include research evidence such as working groups, expert opinions, consensus, or current discourse for the purposes of this review.

Among the 83 included articles, 52 unique critical care triage protocols from 59 articles and 25 ethical frameworks or recommendations from 25 articles were described. Four articles included both a triage protocol and an ethical framework [23-25]. Two articles were computer simulation models and were not assessed as a triage protocol or ethical framework [26, 27]. The 52 included critical care triage protocols have two main approaches: algorithmic (29/52, 55.8\%) or point-based (23/52, 44.2\%) (Supplementary Table 2, Additional File 1). In the algorithmic approach, each patient is assessed for each criterion, which determines if the patient is eligible to receive critical care. For example, the Ontario Health Plan for an Influenza Pandemic (OHPIP) has four main components: inclusion criteria (patients who require critical resources), exclusion criteria (patients who have poor prognosis), minimum qualifications for survival (amount of resources required), and a prioritization tool (color scheme to prioritize patients for ICU admission and mechanical ventilation) [28] (Supplementary Table 2, Additional File 1). Some algorithmic triage protocols are conceptually similar $(7 / 29,24.1 \%)$ (Supplementary Table 2, Additional File 1). Few articles $(10 / 29,34.5 \%)$ [29-37] describe supplemental criteria for situations where triage decisions cannot be reached after evaluating patients based on the criteria. These criteria included fair innings $(5 / 10,50 \%)$, random selection $(3 /$ 10, 30\%), multiplier effect/essential worker/caregiver/ healthcare provider/first come, and first served/groups subjected to social deprivation and disadvantage (each 2/ 10, 20\%) (Supplementary Table 1). The Hamilton Health Adult Critical Care Triage and Resource Allocation Protocol includes additional criteria for situations where 
triage decisions cannot be reached after evaluating patients based on the OHPIP triage protocol (e.g., clinical evidence of a significantly better outcome, multiplier effect which is those with skills and knowledge to save others during the pandemic) [36]. The article by Winsor et al. summarizes common "supplementary criteria" and included a list of key considerations when jurisdictions are considering supplementary triage criteria.

Most included algorithm triage protocols (18/29, 62.1\%) included a Sequential Organ Failure Assessment (SOFA) score as exclusion criterion. Of these, $10(10 / 18$, $55.6 \%)$ proposed a SOFA score of $>11$ to exclude patients from critical care resources [25, 28, 30, 34, 35, 38-42]. Seven did not state a cutoff $[29,31,43-47]$, and one proposed a SOFA score $\geq 14$ or $\leq 8$ [48]. Only four $(4 / 29$, $13.8 \%)$ triage protocols included a criterion for patient age ( $>85$ years) as an exclusion criterion [28, 34, 49, 50]. Seven $(7 / 29,24.1 \%)$ include frailty as a criterion $[29,42,44,46$, 51-53]. Most triage protocols $(19 / 29,65.5 \%)$ include a reassessment and re-categorization of each patient (e.g., at 48 and $120 \mathrm{~h}$ ) to determine if patients are improving and are still eligible for critical care [28-33, 35, 38, 39, 42-44, 46-49, 53, 54].

Twenty-three unique point-based triage protocols were described in the included studies (Supplementary Table 2, Additional File 1). In a point-based triage approach, scores are used to determine who in the casemix of patients should receive critical care resources. Of the 23 point-based triage protocols, eight $(8 / 23,34.5 \%)$ included triage protocols developed for an infectious disease outbreaks [55-66] (AGILITIES, Clinical Dashboard, Community Assessment Tools [CAT], Critical Care Disaster Resource Allocation Framework, Pandemic Modified Warning Score [P-MEWS], Simple Triage Scoring System [STSS], Swine Flu Triage [SWiFT], and XGBoost Machine Learning Algorithm). Six (6/23, 26.1\%) included validated predictors of mortality (CURB-65, Nursing Home-Acquired Pneumonia in the Elderly [NHAP], Predisposition Insult Response and Organ Dysfunction [PIRO-CAP], Pneumonia Severity Index [PSI] and Severity Score for the Elderly with CommunityAcquired Pneumonia) [60, 67-69] or intensive respiratory or vasopressor support (SMART-COP) in patients with community acquired pneumonia. Five $(5 / 23,21.7 \%)$ are commonly used scoring systems for severity of illness in the ICU or to determine if a patient requires critical care intervention and include the Acute Physiology And Chronic Health Evaluation-II/III (APACHE-II/ APACHE-III) [70, 71], Modified Early Warning System (MEWS) [66], SOFA [61, 70-76], quick SOFA (qSOFA) [66], or Simplified Acute Physiology Score (SAPS-II) [71]. Two included a scoring system for severe hypoxemic respiratory failure from acute respiratory distress (Murray score, $\mathrm{PaO}_{2} / \mathrm{FiO}_{2}$ ratio) [63, 77]. One included a predictor of mortality for sepsis (Mortality in Emergency Department Sepsis [MEDS]) [60, 63]. Most pointbased triage protocols included the patient's age (12/23, 52.2\%) (Table 1) that is gender specific (PSI-men: points in years, women: points in years- 10), includes age categories (e.g., 0-49, 50-69, 70-84, $\geq 85$ ), a multiplier (e.g., $0.5 x$ ), or an age cutoff (e.g., $\geq 65, \geq 75, \geq 85$ ). In all cases, the older the patient is, the more points they are assigned (i.e., less favorable). Nearly all point-based triage protocols $(20 / 23,87 \% \%)$ included vital signs, with the most commonly included being respiratory rate (18/ $23,78.3 \%)$, blood pressure $(14 / 23,60.9 \%)$, and heart rate $(12 / 23,52.2 \%)$. Nearly all point-based triage protocols included laboratory or radiographic findings, with oxygenation $(14 / 23,60.9 \% \%)$ the most commonly included criterion. One point-based triage protocol deducted points (i.e., were more favorable) for patients who were pregnant [58].

Sixteen of the included studies described the accuracy of 18 critical care triage protocols (Supplementary Table 3, Additional File 1) to predict mortality (13/16, 81.2\%)(Table 2) [57, 59, 60, 62, 66, 67, 69, 71, 72, 74, 75, $78]$, ICU admission (10/16, 62.5\%), and need for mechanical ventilation $(6 / 16,37.5 \%)$ (Supplementary Table 3, Additional File 1) [61-63, 72, 74]. Four of these studies evaluated the performance of an algorithm-based triage protocol $[76,78,79]$, with three evaluating the OHPIP $[76,78,79]$. Of the patients who met the criteria for OHPIP blue (i.e., palliative care only and would not be admitted to the ICU), $24.6-63.0 \%$ died and $27.3-75.4 \%$ survived $[63,76,78,79]$. Of the patients who met the criteria for OHPIP red (i.e., high priority for ICU admission), 65-93.7\% survived and 6.3-35.0\% died (Table 2). One article used a machine-learning based algorithm, which predicted mortality in all ICU patients (AUROC $0.862)$ more accurately compared to the qSOFA (0.760), MEWS (0.833), and CURB-65 (0.652) [66]. The remaining articles evaluated the performance of pointbased triage protocols. Five articles evaluated four triage protocols developed for an infectious disease outbreak: CAT, P-MEWS, STSS, and SWiFT. No score was a good predictor of mortality with AUCs ranging from 0.58 (95\% CI 0.46-0.69; P-MEWS) to 0.71 (95\% CI 0.66-0.77; STSS) (Supplementary Table 3, Additional File 1). Six included articles evaluated scoring systems for severity of illness in the ICU: APACHE-II/APACHE-III, MEWS, qSOFA/SOFA, and SAPS-II (Supplementary Table 3, Additional File 1). The SOFA score on admission was a good predictor of 30-day mortality in one study with an AUC of 0.83 (95\% CI 0.81-0.85]) [72]. Two studies reported that a SOFA score $>11$ was a fair predictor of mortality (AUC 0.74 and 0.65) [70, 78]. Six studies reported in-hospital mortality in patients with a SOFA score $>11$, which ranged from $0 \%[73]$ to $59 \%$ (95\% CI 
Table 1 Categories of criteria included in published point-based triage protocols

\begin{tabular}{|c|c|c|c|c|c|}
\hline Point-based criteria & Age & Comorbidities & Vital signs & Laboratory findings & Neurological status \\
\hline \multicolumn{6}{|l|}{ Pandemic } \\
\hline AGILITIES Score & $\checkmark$ & $\checkmark$ & $\checkmark^{\mathrm{b}, \mathrm{e}}$ & $\boldsymbol{\checkmark}^{1}$ & $\sqrt{ }(\mathrm{GCS})$ \\
\hline CAT & $x$ & $x$ & $\checkmark^{b}$ & $x$ & $\sqrt{ }(\mathrm{ALOC})$ \\
\hline Clinical dashboard & $\checkmark$ & $\checkmark$ & $\boldsymbol{V}^{\mathrm{a}, \mathrm{b}, \mathrm{c}, \mathrm{e}}$ & $\boldsymbol{\checkmark}^{6,8,11-16}$ & $x$ \\
\hline Critical Care Disaster Resource Allocation & $\checkmark$ & $\checkmark$ & $\boldsymbol{V}^{\mathrm{a}, \mathrm{b}}$ & $\checkmark^{1,9,10}$ & $x$ \\
\hline P-MEWS & $x$ & $x$ & $\checkmark^{a, b, c, d}$ & $x$ & $x$ \\
\hline STSS & $\checkmark$ & $x$ & $\checkmark^{\mathrm{b}}$ & $\checkmark^{1}$ & $\sqrt{ }$ (mental status) \\
\hline SWiFT & $x$ & $x$ & $\checkmark^{a, b, c, d}$ & $\sqrt{ }^{1}$ & $\sqrt{ }$ (mental status) \\
\hline XGBoost Machine Learning Algorithm & $\checkmark$ & $x$ & $\checkmark^{a, b, c, d}$ & $\boldsymbol{\checkmark}^{6,8,10}$ & $x$ \\
\hline \multicolumn{6}{|l|}{ Community-acquired pneumonia } \\
\hline CURB-65 & $\checkmark$ & $x$ & $\boldsymbol{\checkmark}^{\mathrm{a}, \mathrm{b}}$ & $\checkmark^{6}$ & $\sqrt{ }$ (mental status) \\
\hline NHAP & $x$ & $x$ & $\checkmark^{b, c}$ & $x$ & $\sqrt{ }$ (mental status) \\
\hline PIRO-CAP & $\checkmark$ & $\checkmark$ & $x$ & $\boldsymbol{\checkmark}^{1,6}$ & $x$ \\
\hline PSI & $\checkmark$ & $\checkmark$ & $\checkmark^{a, b, c, d}$ & $\mathcal{\checkmark}^{1,2,3,5,6,7}$ & $\sqrt{ }$ (mental status) \\
\hline Severity Score for the Elderly with CAP & $\checkmark$ & $\checkmark$ & $\boldsymbol{V}^{a, b, c, d, e}$ & $\sqrt{6}^{6}$ & $x$ \\
\hline SMART-COP & $x$ & $x$ & $\boldsymbol{V}^{\mathrm{a}, \mathrm{b}, \mathrm{c}}$ & $\mathfrak{\checkmark}^{1}$ & $\sqrt{ }$ (mental status) \\
\hline \multicolumn{6}{|l|}{ ECMO allocation strategy } \\
\hline Minnesota Department of Health & $\checkmark$ & $x$ & $x$ & $\checkmark^{1,9,10}$ & $\sqrt{ }(\mathrm{GCS})$ \\
\hline \multicolumn{6}{|l|}{ ICU scoring systems } \\
\hline APACHE-II & $\checkmark$ & $x$ & $\checkmark^{a, b, c, d}$ & $\checkmark^{1,2,3,4,6,6,7,8}$ & $\sqrt{ }(\mathrm{GCS})$ \\
\hline MEWS & $x$ & $x$ & $\checkmark^{a, b, c, d}$ & $x$ & $\checkmark(A \vee P U)$ \\
\hline qSOFA & $x$ & $x$ & $\boldsymbol{V}^{\mathrm{a}, \mathrm{b}}$ & $x$ & $\sqrt{ }(\mathrm{GCS})$ \\
\hline SAPS-II & $\checkmark$ & $\checkmark$ & $\boldsymbol{V}^{\mathrm{a}, \mathrm{c}, \mathrm{d}}$ & $\checkmark^{1,2,3,4,6,8}$ & $\sqrt{ }(\mathrm{GCS})$ \\
\hline SOFA & $x$ & $x$ & $x$ & $\checkmark^{1,9,10}$ & $\sqrt{ }(\mathrm{GCS})$ \\
\hline \multicolumn{6}{|l|}{ Sepsis } \\
\hline MEDS & $\checkmark$ & $\checkmark$ & $\checkmark^{b}$ & $\sqrt{ }^{8,9}$ & $\sqrt{ }($ mental status $)$ \\
\hline \multicolumn{6}{|l|}{ ARDS } \\
\hline Murray score & $x$ & $x$ & $x$ & $\boldsymbol{J}^{1}$ & $x$ \\
\hline $\mathrm{PaO}_{2} / \mathrm{FiO}_{2}$ ratio & $x$ & $x$ & $x$ & $\boldsymbol{\checkmark}^{1}$ & $x$ \\
\hline
\end{tabular}

Abbreviations: AGILITIES Age, Glasgow score, Infusions, Lungs, Interventions, Tests, Informal/incidental, Excessive weight, Subtract; ALOC altered level of consciousness; APACHE-II Acute Physiology and Chronic Health Evaluation; CAP community-acquired pneumonia; CAT Community Assessment Tools; CURB-65 Confusion, Urea, Respiratory rate, Blood pressure, Age $\geq 65$; GCS Glasgow Coma Scale; MEDS Mortality in Emergency Department Sepsis; MEWS Modified Early Warning Score; NHAP Nursing Home-Acquired Pneumonia in the Elderly; P-MEWS Pandemic Modified Warning Score; PIRO-CAP Predisposition Insult Response and Organ Dysfunction in Community Acquired Pneumonia; PS/ Pneumonia Severity Index; qSOFA quick Sequential Organ Failure Assessment; SAPS-II Simplified Acute Physiology Score; SOFA Sequential Organ Failure Assessment; SMART-COP Systolic blood pressure, Multilobar involvement, Respiratory rate, Tachycardia, Confusion, Oxygenation; STSS Simple Triage Scoring System; SWiFT Swine Flu Triage

Vital signs included: a-blood pressure, b-respiratory rate, c-heart rate, d-temperature, e-height/weight/BM!

Laboratory findings included: 1-oxygenation, 2-arterial pH or HCO3, 3-sodium (serum), 4-potassium (serum), 5-glucose, 6-kidney (creatinine, BUN, urine output), 7hematocrit, 8-WBC, 9-platelet, 10-bilirubin, 11-procalcitonin, 12-D-dimer, 13-C-reactive protein, 14-LDH, 15-troponin, 16-ferritin

56-62\%) [75] (Table 2). Four included articles evaluated performance of predictors of mortality in community acquired pneumonia for triage of patients during a pandemic $[57,60,67,69]$. No score was a good predictor of mortality with AUCs ranging from 0.64 (95\% CI 0.580.71; PIRO-CAP) to 0.78 (95\% CI 0.72-0.83; PSI).

Thirty of the included articles explicitly stated which ethical principles guided the development of the triage criteria. Of these, twelve articles described ethical frameworks $(12 / 30,40 \%)$ and 13 described guiding principles or recommendations $(13 / 30,43.3 \%)$ for triage decisions during a pandemic (Supplementary Table 4, Additional File 1). Four articles used an ethical framework or recommendation to inform the development of a triage protocol [23-25, 32]. Although terminology differed across the studies, they can be divided between substantive values (e.g., duty to provide care, equity, stewardship, etc.) and procedural values (e.g., reasonable, open and transparent, etc.) [80] (Table 3). For example, most included the principle of stewardship $(20 / 30,66.7 \%)$ in 
Table 2 Diagnostic accuracy of triage tools to predict mortality

\begin{tabular}{|c|c|c|c|}
\hline Triage tool (mortality risk) & $\begin{array}{l}\text { Hospital mortality, \% } \\
\text { (per triage tool) }\end{array}$ & $\begin{array}{l}\text { Patients meeting } \\
\text { triage criteria, \% }\end{array}$ & Key operating characteristics \\
\hline \multicolumn{4}{|l|}{ Algorithmic } \\
\hline $\begin{array}{l}\text { Machine Learning Algorithm } \\
\text { (12-h window) [66] }\end{array}$ & 9.6 & NR & $\begin{array}{l}\text { AUC: } 0.75-0.86 \\
\text { Se: } 0.80 \\
\text { Sp: } 0.75\end{array}$ \\
\hline OHPIP blue $[63,76,78,79]$ & 10.9-29.1 & $18.8-27.8$ & $\begin{array}{l}\text { AUC: } 0.816 \\
\text { Se: } 0.76 \\
\text { Sp: } 0.91 \\
\text { TP (\%): } 24.6-63.0 \\
\text { FP (\%): } 27.3-75.4\end{array}$ \\
\hline OHPIP red & NR & $19.8-43.9$ & $\begin{array}{l}\text { AUC/Se/Sp: NR } \\
\text { TN (\%): 65.0-93.7 } \\
\text { FN (\%): 6.3-35.0 }\end{array}$ \\
\hline OHPIP yellow & NR & $4.0-11.4$ & $\begin{array}{l}\text { AUC/Se/Sp: NR } \\
\text { TN (\%): 41.4-75.0 } \\
\text { FN (\%): 25-58.6 }\end{array}$ \\
\hline OHPIP green & NR & $0-65.3$ & $\begin{array}{l}\text { AUC: NR } \\
\text { Se: } 0.66 \\
\text { Sp: } 0.83 \\
\text { TP (\%): } 0-4.5 \\
\text { FP (\%): } 95.5-100\end{array}$ \\
\hline \multicolumn{4}{|l|}{ Point-based (severity of illness) } \\
\hline MSOFA > $11[72]$ & 10.5 & 4.0 & $\begin{array}{l}\text { AUC: } 0.84 \\
\text { Se/Sp: NR } \\
\text { TP (\%): } 57.7 \\
\text { FP (\%): } 42.3\end{array}$ \\
\hline MSOFA 8-11 & NR & 14.6 & $\begin{array}{l}\text { AUC/Se/Sp: NR } \\
\text { TN (\%): } 69.1 \\
\text { FN (\%): } 30.9\end{array}$ \\
\hline qSOFA [66] & 9.6 & NR & $\begin{array}{l}\text { AUC: } 0.76 \\
\text { Se: } 0.95 \\
\text { Sp: } 0.37\end{array}$ \\
\hline MEWS [66] & 9.6 & NR & $\begin{array}{l}\text { AUC: } 0.83 \\
\text { Se: } 0.90 \\
\text { Sp: } 0.56\end{array}$ \\
\hline SOFA > $11[61,70,72,75]$ & $4.8-20.5$ & $5.9-40$ & $\begin{array}{l}\text { AUC: } 0.64-0.85 \\
\text { Se/Sp: NR } \\
\text { TP (\%): 0-59.0 } \\
\text { FP (\%): 41-100 }\end{array}$ \\
\hline SOFA 8-11 & NR & $12.2-44.0$ & $\begin{array}{l}\text { AUC/Se/Sp: NR } \\
\text { TN (\%): } 72.2-83.6 \\
\text { FN (\%): 16.4-27.8 }\end{array}$ \\
\hline \multicolumn{4}{|c|}{ Point-based (epidemic or pandemic) } \\
\hline CAT $\geq 3[57]$ & 6.0 & 18.8 & $\begin{array}{l}\text { AUC: } 0.65 \\
\text { Se: } 0.47 \\
\text { Sp: } 0.83 \\
\text { TN (\%): } 85.2 \\
\text { FN (\%): } 14.8\end{array}$ \\
\hline CAT $<3$ & NR & 81.2 & $\begin{array}{l}\text { AUC/Se/Sp: NR } \\
\text { TP (\%): } 3.9 \\
\text { FP (\%): } 96.0\end{array}$ \\
\hline PMEWS > $3[57,59,60]$ & $6.0-22.6$ & $62.8-70.4$ & $\begin{array}{l}\text { AUC: } 0.60-0.66 \\
\text { Se: } 0.87 \\
\text { Sp: } 0.16 \\
\text { TN (\%): } 74.0-93.4 \\
\text { FN (\%): 6.6-26 }\end{array}$ \\
\hline STSS [60-63] & $4.7-12.3$ & NA & $\begin{array}{l}\text { AUC: } 0.71-0.80 \\
\text { Se/Sp: NR }\end{array}$ \\
\hline
\end{tabular}


Table 2 Diagnostic accuracy of triage tools to predict mortality (Continued)

\begin{tabular}{|c|c|c|c|}
\hline Triage tool (mortality risk) & $\begin{array}{l}\text { Hospital mortality, \% } \\
\text { (per triage tool) }\end{array}$ & $\begin{array}{l}\text { Patients meeting } \\
\text { triage criteria, \% }\end{array}$ & Key operating characteristics \\
\hline STSS 0 & NR & $30.6-44.1$ & $\begin{array}{l}\text { AUC: } 0.71 \\
\text { Se/Sp: NR } \\
\text { TN (\%): } 94.7-99.6 \\
\text { FN (\%): } 0.4-5.3\end{array}$ \\
\hline STSS 1 & NR & $31.7-42.7$ & $\begin{array}{l}\text { AUC/Se/Sp: NR } \\
\text { TN (\%): } 90.1-96.4 \\
\text { FN (\%): } 3.6-9.9\end{array}$ \\
\hline STSS 2 & NR & $14.8-23.8$ & $\begin{array}{l}\text { AUC/Se/Sp: NR } \\
\text { TN (\%): 70.8-84.6 } \\
\text { FN (\%): } 15.4-29.2\end{array}$ \\
\hline STSS $\geq 3$ & NR & $5.9-14.5$ & $\begin{array}{l}\text { AUC/Se/Sp: NR } \\
\text { TN (\%): } 63.8-89.9 \\
\text { FN (\%): } 11.1-36.2\end{array}$ \\
\hline SwiFT [55] & NR & NR & $\begin{array}{l}\text { AUC: } 0.77 \\
\text { Se/Sp: NR }\end{array}$ \\
\hline \multicolumn{4}{|l|}{ Point-based (sepsis) } \\
\hline MEDS > $5[60,64]$ & $10.9-12.0$ & NR & $\begin{array}{l}\text { AUC: } 0.77 \\
\text { Se: } 0.85 \\
\text { Sp: } 0.40\end{array}$ \\
\hline MEDS $\leq 5$ & NR & NR & $\begin{array}{l}\text { AUC/Se/Sp: NR } \\
\text { TP (\%): } 4.8 \\
\text { FP (\%): } 95.2\end{array}$ \\
\hline \multicolumn{4}{|c|}{ Point-based (community acquired pneumonia) } \\
\hline CAP [57] & 11.7 & NR & $\begin{array}{l}\text { AUC: } 0.66 \\
\text { Se/Sp: NR }\end{array}$ \\
\hline CURB-65 $[57,59,60,66-69]$ & $6.0-31.7$ & NR & $\begin{array}{l}\text { AUC: } 0.65-0.79 \\
\text { Se: } 0.97-0.98 \\
\text { Sp: } 0.16-0.18\end{array}$ \\
\hline CURB-65 0-1 (low) & NR & $36.9-37.6$ & $\begin{array}{l}\text { AUC: } 0.67 \\
\text { Se: } 0.94 \\
\text { Sp: } 0.17 \\
\text { TP (\%): } 0-22.1 \\
\text { FP (\%): } 77.9-100\end{array}$ \\
\hline CURB-65 2 (moderate) & NR & 18.1 & $\begin{array}{l}\text { AUC: } 0.64 \\
\text { Se: } 0.48-0.73 \\
\text { Sp: } 0.50-0.78 \\
\text { TN (\%): } 66.7-88.3 \\
\text { FN (\%): } 11.7-33.3\end{array}$ \\
\hline CURB-65 $\geq 3$ (severe) & NR & $0.1-4.6$ & $\begin{array}{l}\text { AUC: } 0.54 \\
\text { Se: } 0-0.36 \\
\text { Sp: } 0.84-0.99 \\
\text { TN (\%): } 33.3-100 \\
\text { FN (\%): } 0-66.7\end{array}$ \\
\hline NHAP [60] & NR & NR & $\begin{array}{l}\text { AUC: } 0.68 \\
\text { Se/Sp: NR }\end{array}$ \\
\hline PIRO-CAP [69] & 31.7 & NR & $\begin{array}{l}\text { AUC: } 0.64 \\
\text { Se/Sp: NR }\end{array}$ \\
\hline PIRO-CAP 0-2 (low) & NR & 27.5 & $\begin{array}{l}\text { AUC: NR } \\
\text { Sen 0.94-0.96 } \\
\text { Sp: 0.07-17.1 } \\
\text { TN (\%): } 86.3 \\
\text { FN (\%): } 13.7\end{array}$ \\
\hline PIRO-CAP 3 (moderate) & NR & 29.1 & $\begin{array}{l}\text { AUC: NR } \\
\text { Se: } 0.88 \\
\text { Sp: } 0.35 \\
\text { TN (\%): } 67.5 \\
\text { FN (\%): } 32.5\end{array}$ \\
\hline
\end{tabular}


Table 2 Diagnostic accuracy of triage tools to predict mortality (Continued)

\begin{tabular}{|c|c|c|c|}
\hline Triage tool (mortality risk) & $\begin{array}{l}\text { Hospital mortality, \% } \\
\text { (per triage tool) }\end{array}$ & $\begin{array}{l}\text { Patients meeting } \\
\text { triage criteria, \% }\end{array}$ & Key operating characteristics \\
\hline PIRO-CAP 4 (high) & NR & 25.3 & $\begin{array}{l}\text { AUC: NR } \\
\text { Se: } 0.58 \\
\text { Sp: } 0.63 \\
\text { TN (\%): } 59.7 \\
\text { FN (\%): } 40.3\end{array}$ \\
\hline PIRO-CAP $\geq 5$ (very high) & NR & 18.1 & $\begin{array}{l}\text { AUC: NR } \\
\text { Se: } 0.01-0.26 \\
\text { Sp: } 0.86-0.99 \\
\text { TN (\%): } 54.2 \\
\text { FN (\%): } 45.8\end{array}$ \\
\hline PSI > 87 [60, 67-69] (low) & $12.0-31.7$ & $41.9-64.0$ & $\begin{array}{l}\text { AUC: } 0.73-0.78 \\
\text { Se: } 46.4-92.9 \\
\text { Sp: } 35.4-80.1 \\
\text { TP (\%): } 13.5-17.4 \\
\text { FP (\%): } 82.6-86.5 \\
\text { TN (\%): } 55.2-97.7 \\
\text { FN (\%): } 2.3-44.8\end{array}$ \\
\hline PSI $\leq 87$ (high) & NR & NR & $\begin{array}{l}\text { AUC: NR } \\
\text { Se: } 93 \\
\text { Sp: } 39\end{array}$ \\
\hline SMRT-CO(P) $[60,67]$ & NR & NR & $\begin{array}{l}\text { AUC: } 0.69 \\
\text { Se/Sp: NR }\end{array}$ \\
\hline SMRT-CO(P) 0-2 (low) & 11.7 & $11-39$ & AUC/Se/Sp: NR \\
\hline SMRT-CO(P) 3 (moderate) & $N R$ & 17 & $\mathrm{~A} \cup \mathrm{C} / \mathrm{Se} / \mathrm{Sp}: \mathrm{NR}$ \\
\hline SMRT-CO(P) $\geq 4$ (high) & NR & 11 & AUC/Se/Sp: NR \\
\hline
\end{tabular}

Abbreviations: AUC area under the curve; Se sensitivity; Sp specificity; TP true positive; $F P$ false positive; $T N$ true negative; $F N$ false negative; $C A P$ Severity Score for the Elderly with Community-Acquired Pneumonia; CATS Community Assessment Tools; Cl confidence interval; CURB-65 Confusion, Urea, Respiratory rate, Blood pressure, age $\geq 65 ;$ MEDS Mortality in Emergency Department Sepsis; MSOFA modified Sepsis-related Organ Failure Assessment; NA not applicable; NHAP Nursing Home-Acquired Pneumonia in the Elderly; NR not reported; PMEWS Pandemic Medical Early Warning Score; OHPIP Ontario Health Plan for an Influenza Epidemic; PIRO-CAP Predisposition, Insult, Response, and Organ Dysfunction in Community-Acquired Pneumonia; PSI Pneumonia Severity Index; SMART-COP or SMRT-CO Systolic blood pressure, Multilobar involvement, Respiratory rate, Tachycardia, Confusion, Oxygenation; SOFA Sepsis-related Organ Failure Assessment; STSS Simple Triage Scoring System; SWiFT Swine Flu Triage

their criteria [10, 24, 35, 83, 85-88, 90, 92-94, 97-100]. This included the duty to steward scarce resources through the principle of utilitarianism (i.e., helping the greatest number of patients to survive the pandemic). Several ethical frameworks or guiding principles included the procedural value of transparency, with 14 $(14 / 30,46.7 \%)$ recommending that triage criteria be disclosed to the public or developed with input from the public $[10,11,23,29,32,35,37,46,82,84,85,88,92$, 93, 95, 98]. Four studies [88, 93, 95, 98] engaged the public to prioritize triage criteria. In all studies, the criterion "most likely to survive" was perceived as fair or was prioritized. The criterion "first come, first served" was perceived as unfair or prioritized in the bottom half of the triage criteria $[32,58,87,88,98]$. Only five studies $(5 / 30,16.7 \%)$ supported allocation of resources based on societal contributions or reciprocity [10, 88, 90, 93, 98].

Several included papers described when triage criteria should be initiated, who should administer the triage criteria, an appeals process, if load leveling is possible to distribute patients throughout a region and consider vulnerable populations. Most included articles suggested that triage criteria be initiated when critical care resources have been overwhelmed despite all efforts to expand critical care capacity to meet demands [10, 11, $25,29,31,32,36,40,44,46,47,50,53,58,82-85,89$, $90,92,98,101]$. Some articles suggested that triage criteria be administered by a triage officer who is an intensivist or other physician with appropriate critical care experience and has no direct contact with the patient $[10,11,33,35,37,38,79,81,85,89]$. Several articles suggested a triage team or committee to remove the burden of the decision from a single individual [23, 25, $29-32,34-36,47,51,52,65,83,93,102]$. This triage team may be important when decisions using supplementary criteria are involved $[34,103]$ or if there are appeals from family or clinicians [23, 24, 92, 93]. This triage team is recommended to include a senior ICU physician, non-physician ICU healthcare professional, and a professional from outside the ICU. This triage process may be overseen by a triage review board [99] or include prospective (i.e., during an appeals process) or retrospective review to ensure accountability, consistent application of the triage criteria, and an adequate level of prioritization (i.e., not over or under triaging) [11, 33, $34,42,89]$. Several articles suggested that an appeals 
Table 3 Substantive and procedural values in published ethical frameworks and guiding principles for critical care triage during a pandemic $(n=30)$

\begin{tabular}{ll}
\hline Substantive values & Example \\
Distributive justice or fairness & $\begin{array}{l}\text { Fairness in how resources are allocated across } \\
\text { members of a group. }\end{array}$ \\
& \\
Duty to plan & Planning for the management of ethical issues \\
& that may arise. \\
"Pulliative care protocol (when patient does not \\
qualify for critical care allocation)."; "The concept \\
of triage by a senior clinician(s) without direct \\
clinical obligation and a support system to \\
implement and manage the triage process." \\
"Triage decisions will not be based on race, \\
Equality \\
ethnicity, gender, disability, insurance status, \\
immigration status, social class, or other \\
non-clinical factors." \\
"Triage decisions during the epidemic should \\
apply to all patients who may require intensive \\
care, not just CoviD-19 patients."
\end{tabular}

$n$ (\%) Author (year published)

8 (26.7) Farrell (2020) [81]; Han (2020) [35]; Leclerc (2020) [42]; Seethala and Keller (2020) [82]; Rhodes (2020) [83]; Steinberg (2020) [84]; Vergano (2020) [53]; Vincent and Creteur (2020) [50]

2 (6.7) Han (2020) [35]; Committee (2020) [47]

13 (43.3) Christian (2010) [85]; Christian (2014) [11]; Cinti (2009) [86]; Daugherty Biddison (2014) [87, 88]; Devereaux (2008) [89]; Eastman (2010) [90]; Einav (2014) [91]; Han (2020) [35]; Herrerros (2020); Lin and Anderson-Shaw (2009) [23]; Powell (2008) [24]; Rubinson (2005) [92]; Silva (2012) [93]

4 (13.3) Marckmann (2020) [46]; Montgomery (2020) [52]; Rhodes (2020) [83]; Steinberg (2020) [84]

17 (56.7) Cheung (2017) [94, 95]; Christian (2010) [85]; Christian (2014) [11]; Cinti (2009) [86]; Devereaux (2008) [89]; Dries (2014) [96]; Daugherty Biddison (2014) [87, 88]; Eastman (2010) [90]; Emanuel [10]; Herrerros (2020); Leclerc (2020) [42]; Powel (2008) [24]; Tillyard (2010) [97]; Silva (2012) [93]; Real de Asua (2020) [32]; Rhodes (2020) [83]; Steinberg (2020) [84]

Reciprocity

Resources allocated based on societal contributions (e.g., caregivers, healthcare providers - feelings of reciprocity).

Stewardship

"Triage decisions regarding the provision of critical care should be guided by the principle of seeking to help the greatest number of people survive the crisis."

5 (16.7) Daugherty Biddison (2014) [87, 88]; Daugherty Biddison (2018) [98]; Eastman (2010) [90]; Emanuel [10]; Silva (2012) [93]

20 (66.7) Cheung (2017) [94, 95]; Christian (2010) [85]; Christian (2014) [11]; Cinti (2009) [86]; Daugherty Biddison (2014) [87, 88]; Daugherty Biddison (2014) [87, 88]; Daugherty Biddison (2018) [98]; Eastman (2010) [90]; Emanual (2020); Han (2020) [35]; Janig (2020) [30]; Marckmann (2020) [46]; Powell (2008) [24]; Rubinson (2005) [92]; Silva (2012) [93]; Steinberg (2020) [84]; Tabery and Mackett (2008) [99]; Real de Asua (2020) [32]; Rhodes (2020) [83]; Tillyard (2010) [97]

Trust "Review of triage decisions (daily retrospective review)."

10 (33.3) Christian (2014) [11]; Chung (2017); Cinti (2009) [86]; Daugherty Biddison (2014) [87, 88]; Daugherty Biddison (2018) [98]; Lin and Anderson-Shaw (2009) [23]; Powell (2008) [24]; Rubinson (2005) [92]; Silva (2012) [93]; Tabery and Mackett (2008) [99]

\section{Procedural values}

Reasonable

Critical care resources be allocated based on specific triage criteria, irrespective of whether the need for resources is related to the current disaster/pandemic or an unrelated critical illness or injury.

Open and transparent

"The criteria for triage should be transparent, public, and as shared as possible. The triage process is the responsibility of the entire society."
12 (40) Cheung (2017) [94, 95]; Christian (2010) [85]; Christian (2014) [11]; Daugherty Biddison (2014) [87, 88]; Devereaux [89]; Dries (2014) [96]; Eastman (2010) [90]; Einav (2014) [91]; Lin and Anderson-Shaw (2009) [23]; Powell (2008) [24]; Rubinson (2005) [92]; Silva (2012) [93]

14 (46.7) Cheung (2017) [94, 95]; Christian (2010) [85]; Christian (2014) [11]; Daugherty Biddison (2014) [87, 88]; Daugherty Biddison (2018) [98]; Han (2020) [35]; Lin and Anderson-Shaw (2009) [23]; Marckmann (2020) [46]; Real de Asua (2020) [32]; Rubinson (2005) [92]; Silva (2012) [93]; Seethala and Keller (2020) [82]; Steinberg 
Table 3 Substantive and procedural values in published ethical frameworks and guiding principles for critical care triage during a pandemic $(n=30)$ (Continued)

\begin{tabular}{|c|c|c|c|}
\hline & Example & $n(\%)$ & Author (year published) \\
\hline & & & (2020) [84]; Valiani (2020) [29] \\
\hline Inclusive & $\begin{array}{l}\text { "Hospitals should establish procedure in advance } \\
\text { of a crisis. These protocols should be developed } \\
\text { regionally and with input from stakeholders } \\
\text { (including the public)." }\end{array}$ & $7(23.3)$ & $\begin{array}{l}\text { Cheung (2017) [94, 95]; Christian } \\
\text { (2010) [85]; Christian (2014) [11]; } \\
\text { Daugherty Biddison (2014) [87, 88]; } \\
\text { Daugherty Biddison (2018) [98]; } \\
\text { Rubinson (2005) [92]; Silva (2012) [93] }\end{array}$ \\
\hline Responsive & $\begin{array}{l}\text { "Prioritization guidelines should differ by } \\
\text { intervention and should respond to changing } \\
\text { scientific evidence." }\end{array}$ & $4(13.3)$ & $\begin{array}{l}\text { Devereaux (2008) [89]; Emmanuael } \\
\text { (2020); Real de Asua (2020) [32]; } \\
\text { Valuani (2020) }\end{array}$ \\
\hline Accountable & $\begin{array}{l}\text { "Triage Review Board-to oversee switch from } \\
\text { traditional ethics of individual autonomy } \\
\text { to an ethics of public health." }\end{array}$ & $5(16.7)$ & $\begin{array}{l}\text { Christian (2014) [1 1]; Lin and } \\
\text { Anderson-Shaw (2009) [23]; } \\
\text { Seethala and Keller (2020) [82]; } \\
\text { Tabery and Mackett (2008) [99]; } \\
\text { Valiani (2020) [29] }\end{array}$ \\
\hline
\end{tabular}

process is not feasible given time and resource constraints [24, 36, 89]. Some articles recommend including an appeal process, but limiting appeals to deviations from the triage process or reevaluations due to updated clinical information [10, 11]. Articles discussed a regionalization scheme for highly sophisticated critical care (e.g., ECMO) [77] or transferring patients to less strained ICUs $[38,42]$. When withdrawing critical care from one patient to reallocate it to another patient with a better prognosis [98], articles outline the ethical [24] and legal challenges associated [90]. Some articles described the importance of educating the public on triage criteria and early family involvement in decision-making as a way to potentially mitigate some of the issues raised by families when decisions to withhold care are made $[23,24,92,93]$. Several articles advocated for the consideration of vulnerable populations [96] to ensure that medical treatment in the context of a pandemic is not restricted to those able to pay [10] or that allocation decisions do not replicating existing inequities (e.g., insured vs. uninsured, urban vs. rural) [98]. Moreover, secondary triage factors may be necessary for selecting among patients with similar prognosis. In this case, the fairest option in the included articles was randomization versus first-come first-served, which penalized people of lower socio-economic means or minority populations $[10,35]$. Several articles recommend the use of advanced age as triage criterion [45], while others reject the use of age and instead encourage frailty, which takes into consideration functional and cognitive status and burden of comorbidities [35, 42, 44, 50, 81].

\section{Quality analysis}

Of 83 included articles, 80 were evaluated for quality (Table 4). Three articles could not be assessed because we could not find quality criteria for evaluating studies that included a computer-generated study population or development of an intelligence dashboard [26, 27, 65]. Five included studies were published conference abstracts and, as such, we did not have enough methodological information to determine their risk of bias. The remaining 75 full-text articles were deemed of sufficient quality to include in analyses (Table 4).

\section{Discussion}

The current study summarizes the literature from 83 studies that described triage criteria created before, during, and after infectious disease outbreaks (e.g., H1N1, SARS, H5N1, MERS, Ebola, COVID-19). Of the 52 triage criteria from 60 articles, 29 were algorithmic-based and 23 were point-based. There were 30 articles that describe ethical frameworks or guiding principles for triage decisions. Most of the algorithm-based triage criteria are based on the OHPIP triage protocol for critical care [28] or included a SOFA score when making triage decisions. Most point-based triage protocols consider the patient's age or vital signs in the total score. Most triage criteria have not been validated and the operating characteristics for those that have (e.g., SOFA) to predict mortality are modest.

It is expected that triage protocols should perform better than first-come, first served distribution of resources as a means to allocate scarce resources and maximize patient survival [27]. However, the results of this rapid review suggest that most protocols are not validated or do not have sufficient criterion validity to predict mortality. Validated scoring systems such as a SOFA or APACHE-II score or pneumonia scoring systems were designed for a specific population and have been demonstrated to be less accurate when used in other patient populations [97, 104-108]. A recent systematic review on prediction models for diagnosis and prognosis of COVID-19 reported that proposed models were poorly described, had a high risk of bias, and may be unreliable 
Table 4 Quality analysis of included articles

\begin{tabular}{|c|c|c|c|}
\hline Author (year) & Critical appraisal tool & $\begin{array}{l}\text { Criteria met, } \\
\text { n/total (\%) }\end{array}$ & $\begin{array}{l}\text { Overall appraisal } \\
\text { (reason) }\end{array}$ \\
\hline Adalja (2013) [77] & JBl-text \& opinion & $5 / 6(83.3)^{*}$ & Include \\
\hline Adeniji and Cusack (2011) & JBI-cohort & $7 / 11(63.6)$ & Include \\
\hline Ardagh (2006) [101] & JBl-text \& opinion & $3 / 6(50.0)$ & Include \\
\hline Ashton-Cleary (2011) [40] & JBl-cross-sectional & $6 / 8(75.0)$ & Include \\
\hline Azoulay (2020) [44] & JBl-text \& opinion & $6 / 6(100)$ & Include \\
\hline Barie (2020) [124] & JBl-text \& opinion & $5 / 6(83.3)$ & Include \\
\hline Brandao-Neto [125] & JBI-cohort & $7 / 11(63.6)^{*}$ & Include \\
\hline Challen (2007) & JBI-cohort & $8 / 11(72.7)$ & Include \\
\hline Cheung (2012)-MJA [39] & JBI-cohort & 9/11 (81.8) & Include \\
\hline Cheung (2012)-Crit Care Resusc [48] & JBI-cohort & 9/11 (81.8) & Include \\
\hline Cheung (2017) [94, 95] & JBI-cross-sectional & $6 / 8(75.0)$ & Include \\
\hline Christian (2006) [28] & JBl-text \& opinion & $5 / 6(83.3)^{*}$ & Include \\
\hline Christian (2009) [79] & JBI-cohort & 8/11 (72.7) & Include \\
\hline Christian (2010) [85] & Delphi process & $5 / 11(45.5)$ & Include \\
\hline Christian (2014) [11] & Delphi process & 10/11 (90.9) & Include \\
\hline Cinti (2009) [86] & JBl-text \& opinion & $6 / 6(100)$ & Include \\
\hline Commons and Denholm (2012) [67] & JBI-diagnostic test accuracy & $5 / 10(50.0)$ & Include \\
\hline Daugherty Biddison (2014)-CHEST [88] & Delphi process & 10/11 (90.9) & Include \\
\hline Daugherty Biddison (2014)-Annals ATS [87] & $\begin{array}{l}\text { JBI-qualitative } \\
\text { JBI-quasi-experimental }\end{array}$ & $\begin{array}{l}6 / 10(60.0) \\
5 / 9(55.6)^{*}\end{array}$ & Include \\
\hline Daugherty Biddison (2018) [98] & $\begin{array}{l}\text { JBI-qualitative } \\
\text { JBI-quasi-experimental }\end{array}$ & $\begin{array}{l}8 / 10(80.0) \\
5 / 9(55.6)^{*}\end{array}$ & Include \\
\hline Daugherty Biddison (2019) [58] & JBl-text \& opinion & $5 / 6(83.3)^{*}$ & Include \\
\hline Devereaux (2008) [89] & JBl-text \& opinion & $5 / 6(83.3)^{*}$ & Include \\
\hline Dries (2014) [96] & Delphi process & 10/11 (90.9) & Include \\
\hline Eastman (2010) [90] & JBl-text \& opinion & $6 / 6(100)$ & Include \\
\hline Ehmann (2020) [31] & JBl-text \& opinion & $6 / 6(100)$ & Include \\
\hline Einav (2014) [91] & Delphi process & 10/11 (90.9) & Include \\
\hline Emanuel [10] & JBl-text \& opinion & $5 / 6(83.3)^{*}$ & Include \\
\hline Enfield (2011) [70] & JBl-cohort & $6 / 11(54.5)$ & $\begin{array}{l}\text { Seek further info } \\
\text { (conference abstract) }\end{array}$ \\
\hline Estella (2012) [68] & JBI-cohort & 9/11 (81.8) & Include \\
\hline Farrell (2020) [81] & JBl-text \& opinion & $6 / 6(100)$ & Include \\
\hline Frolic (2009) [36] & AGREE Reporting Checklist & $14 / 23(60.9)$ & Include \\
\hline Grissom (2010) [72] & JBI-cohort & 9/11 (81.8) & Include \\
\hline Guest (2009) [78] & JBl-cohort & $7 / 11(63.6)$ & Include \\
\hline Gupta (2020) [51] & JBl-text \& opinion & $5 / 6(83.3)$ & Include \\
\hline $\operatorname{Han}(2020)$ [35] & JBl-text \& opinion & $6 / 6(100)$ & Include \\
\hline Herreros (2020) [45] & JBl-text \& opinion & $6 / 6(100)$ & Include \\
\hline Hick (2006) [43] & JBl-text \& opinion & $5 / 6(83.3)^{*}$ & Include \\
\hline Ibrahim (2020) [65] & $\begin{array}{l}\text { No critical appraisal for development } \\
\text { of intelligence dashboard }\end{array}$ & Not applicable & Include \\
\hline Janig (2020) [30] & JBl-text \& opinion & $6 / 6(100)$ & Include \\
\hline Kanter (2015) [27] & No critical appraisal for modeling data & Not applicable & Include \\
\hline Kaposy (2010) [34] & JBl-text \& opinion & $5 / 6(83.3)$ & Include \\
\hline Khan (2009) [73] & JBI-cohort & $7 / 11(63.6)$ & Include \\
\hline Leclerc (2020) [42] & JBI-text \& opinion & $6 / 6(100)$ & Include \\
\hline
\end{tabular}


Table 4 Quality analysis of included articles (Continued)

\begin{tabular}{|c|c|c|c|}
\hline Author (year) & Critical appraisal tool & $\begin{array}{l}\text { Criteria met, } \\
\text { n/total (\%) }\end{array}$ & $\begin{array}{l}\text { Overall appraisal } \\
\text { (reason) }\end{array}$ \\
\hline Lin and Anderson-Shaw (2009) [23] & JBl-text \& opinion & $5 / 6(83.3)^{*}$ & Include \\
\hline Marckmann (2020) [46] & JBl-text \& opinion & 6/6 (100) & Include \\
\hline Marriott (2012) [41] & JBI-cohort & $6 / 11(54.5)$ & $\begin{array}{l}\text { Seek further info } \\
\text { (conference abstract) }\end{array}$ \\
\hline Miller (2010) [74] & JBI-cohort & $7 / 11(63.6)$ & $\begin{array}{l}\text { Seek further info } \\
\text { (conference abstract) }\end{array}$ \\
\hline Montgomery (2020) [52] & JBl-text \& opinion & $4 / 6(66.7)^{*}$ & Include \\
\hline Morton (2014) [63] & JBI-cohort & $8 / 11(72.7)$ & $\begin{array}{l}\text { Seek further info } \\
\text { (conference abstract) }\end{array}$ \\
\hline Morton (2015) [65] & JBI-cohort & 8/11 (72.7) & Include \\
\hline Muller (2010) [60] & JBI-cohort & 9/11 (81.8) & Include \\
\hline Murad (2009) [54] & JBl-cross-sectional & 4/8 (50.0) & Include \\
\hline Myles (2012) [57] & JBI-case control & $9 / 10(90.0)$ & Include \\
\hline PCP Ethic Committee (2020) [47] & JBl-text \& opinion & $3 / 6(50.0)$ & Include \\
\hline Pereira (2012) [69] & JBl-cohort & 9/11 (81.8) & Include \\
\hline Powell (2008) [24] & AGREE Reporting Checklist & $12 / 23(52.2)$ & Include \\
\hline Prekker (2020) [25] & JBl-text \& opinion & $5 / 6(83.3)^{*}$ & Include \\
\hline Real de Asua (2020) [32] & JBl-text \& opinion & 5/6 (83.3) & Include \\
\hline Rhodes (2020) [83] & JBl-text \& opinion & $5 / 6(83.3)$ & Include \\
\hline Rowan (2010) [55] & JBI-cohort & $8 / 11(72.7)$ & Include \\
\hline Rubinson (2005) [92] & JBl-text \& opinion & $4 / 6(66.7)^{*}$ & Include \\
\hline Ryan (2020) [66] & JBI-cohort & $7 / 11(63.6)$ & Include \\
\hline Saleh (2016) [71] & JBI-cohort & $6 / 11(54.5)$ & $\begin{array}{l}\text { Seek further info } \\
\text { (conference abstract) }\end{array}$ \\
\hline Seethala and Keller (2020) [82] & JBl-text \& opinion & $6 / 6(100)$ & Include \\
\hline Shahpori (2011) [75] & JBI-cohort & 10/11 (90.9) & Include \\
\hline Shekar (2020) [102] & JBl-text \& opinion & $6 / 6(100)$ & Include \\
\hline Silva (2012) [93] & JBI-qualitative & $8 / 10(80.0)$ & Include \\
\hline Sprung (2010) [38] & Delphi process & $6 / 11(54.5)$ & Include \\
\hline Sprung (2020) [33] & JBl-text \& opinion & 5/6 (83.3) & Include \\
\hline Steinberg (2020) [84] & JBl-text \& opinion & 6/6 (100) & Include \\
\hline $\begin{array}{l}\text { Swiss Academy of Medical Sciences \& Swiss } \\
\text { Society of Intensive Care (2020) [49] }\end{array}$ & AGREE Reporting Checklist & $12 / 23(52.2)$ & Include \\
\hline Tabery and Mackett (2008) [99] & JBl-text \& opinion & $6 / 6(100)$ & Include \\
\hline Talmor (2007) [62] & JBI-cohort & 10/11 (90.9) & Include \\
\hline Tambone (2020) [107] & JBl-text \& opinion & $4 / 6(66.7)$ & Include \\
\hline Tillyard (2010) [97] & JBl-text \& opinion & $5 / 6(83.3)$ & Include \\
\hline Utley (2011) [26] & $\begin{array}{l}\text { No critical appraisal for } \\
\text { modeling data }\end{array}$ & Not applicable & Include \\
\hline Valiani (2020) [29] & JBl-text \& opinion & $6 / 6(100)$ & Include \\
\hline Vergano (2020) [53] & JBl-text \& opinion & $4 / 6(66.7)$ & Include \\
\hline Vincent and Creteur (2020) [50] & JBl-text \& opinion & 2/6 (33.3) & Include \\
\hline Warrillow (2020) [37] & JBl-text \& opinion & $5 / 6(83.3)$ & Include \\
\hline Wilkens and Klein (2010) [56] & JBl-cross-sectional & 4/8 (50.0) & Include \\
\hline Williams and Gannon (2009) [76] & JBI-cohort & $7 / 11(63.6)$ & Include \\
\hline Winsor (2014) [103] & JBl-text \& opinion & $6 / 6(100)$ & Include \\
\hline
\end{tabular}


when used in situ [109]. Most triage protocols were developed with experts (e.g., ethicists, lawyers, healthcare professionals, decision-makers and, in some cases, members of the public) in advance of a pandemic (e.g., CHES $T$ consensus statements) $[11,88,91,96,100,110]$ or were validated using a non-representative population (e.g., patients with COVID-19, seasonal influenza or ARDS) [111-119]. As such, most triage criteria are not validated (and it may not be possible to validate them) prior to their use. Our study adds to the existing reviews that discuss implementation of triage plans [12], ethics of triage [14], ventilator triage policies [15], and quality of criteria to triage and transfer critically ill patients [13] by providing decision-makers with data to help them select and tailor tools best for their jurisdictions.

In general, triage is grounded by utilitarian theory or "the greatest good for the greatest number" or egalitarianism or "equal distribution of resources." Both considerations are warranted during an infectious disease outbreak as triage criteria should benefit society (utilitarian) but ensure fairness (egalitarian). As such, health systems should weigh ethical principles and decide which core values should underpin the triage criteria. In addition to identifying patients at a high risk of mortality (e.g., 80-90\%), triage protocols should also separate patients into cohorts of relative prognosis (e.g., 40-50$60 \%$ ) with frequent revaluation in order to apply a utilitarian framework, as opposed to queuing or randomization. This rapid review suggests that most triage criteria were developed by a panel of experts and few sought public input. Triage criteria should be anchored by the procedural value of open and transparent and the substantive value of trust by engaging the public in triage development and educating the public on triage during a pandemic. In some cases, triage criteria may include the substantive value of reciprocity. For example, the developers of the Newfoundland and Labrador Critical Care Triage protocol [34] considered Indigenous populations where the death of an elder may have a devastating impact on this community. Low-income countries (e.g., South Sudan) have a shortage of skilled critical care healthcare professionals and, as such, these healthcare professionals should be given priority for receiving scarce resources because they could assist in the care of critically ill patients after they recover [120]. Health systems need to pick triage tools that consider cultural values and ideologies while adhering to utilitarian and egalitarian principles.

The large number of patients admitted to ICU during the COVID-19 pandemic provides an opportunity to validate (retrospectively and prospectively), improve, and standardize selected triage tools. This may include evaluating how well they work as prognostic models to predict mortality and length of ICU stay or length of mechanical ventilation (i.e., utilization of resources) or the ability for each criterion or a combination of criteria identified by this rapid review (e.g., age, sex, comorbidities, vital signs, SOFA score) to act as proxies for outcomes. This is important given the uncertainty about how the current pandemic will evolve and when future pandemics will occur. This should include engaging the public to ensure that triage criteria represent societal values as well as to inform members of the public how decisions will be made $[87,92,93,98,100]$.

There are several strengths of our rapid review. The timely synthesis of published triage criteria is important for health systems to respond to the current pandemic and prepare for future pandemics and for evidence to be generated on the utility of the currently available triage criteria to allocate scarce resources and maximize survival. The rapid review followed rigorous methodology [121] that included a search strategy created with experts, a comprehensive literature review, and all steps (full-text screening, data extraction and quality assessments) completed independently and in duplicate. There are several limitations of our rapid review that should be considered. This includes the possibility that some studies were missed in the search, during selection of studies, or because of the rapidly evolving COVID-19 literature. This also includes the exclusion of unpublished triage criteria that is available on institutional, government or society websites though most of these triage criteria either are adapted from or cite triage criteria included in this review. The current study provides a catalog of possible criteria, but with only limited data on which criteria work best. Included studies were largely developed outside of the scope of pandemics or infectious disease outbreaks, as it is difficult to know how they will perform during an infectious disease outbreak. Additionally, our review is focused on an adult critical care setting as pediatric populations use different types of triage criteria. Resource allocation and triage in pediatric groups has been explored elsewhere [122, 123]. Regardless of the operating characteristics, healthcare systems need to decide which triage protocol to enact if the demand of critical care resources exceeds supply to ensure a standardized and ethically sound approach to allocating health resources.

\section{Conclusion}

This rapid review summarizes the existing published triage criteria used for epidemics or pandemics when ICU resources are scarce. Despite the limited validation of criteria and the modest operating characteristics of those criteria that have been validated, our study provides healthcare decision-makers with a list of available criteria, their elements, and their operating characteristics. Given the uncertainty of how the current pandemic will 
evolve and when future pandemics occur, all healthcare systems need to pick the criteria that work best for their circumstances. The triage process needs to adhere to utilitarian and egalitarian principles. Moreover, public engagement is key to ensure that triage criteria represent societal values and to ensure that members of the public understand how decisions will be made. Health systems and jurisdictions should validate their chosen triage criteria during the current COVID-19 pandemic to develop a new model of triage in preparation for the current and future pandemics.

\section{Supplementary Information}

The online version contains supplementary material available at https://doi. org/10.1186/s12916-020-01871-9.

Additional file 1: Supplementary Appendix 1. (PRISMA checklist for systematic reviews), Supplementary Appendix 2. (MEDLINE search strategy), Supplementary Table 1. (Study characteristics),

Supplementary Table 2. (Triage protocols based on an algorithm or point-based method), Supplementary Table 3. (Diagnostic accuracies of triage criteria), and Supplementary Table 4. (Ethical frameworks or guiding principles for triage decisions during a pandemic).

\section{Abbreviations}

APACHE-II or APACHE-III: Acute Physiology and Chronic Health Evaluation II: CAT: Community Assessment Tools; COVID-19: Novel coronavirus SARS-CoV2; ICU: Intensive care unit; MEDS: Mortality in Emergency Department Sepsis; NHAP: Nursing Home-Acquired Pneumonia in the Elderly; OHPIP: Ontario Health Plan for an Influenza Pandemic; P-MEWS: Pandemic Modified Warning Score; PIRO-CAP: Predisposition Insult Response and Organ Dysfunction; PSI: Pneumonia Severity Index; SOFA: Sequential Organ Failure Assessment; STSS: Simple Triage Scoring System; SAPS-II: Simplified Acute Physiology Score; SWiFT: Swine Flu Triage

\section{Acknowledgements}

The authors would like to thank Nicole Dunnewold (University of Calgary) with their help reviewing the search strategies.

\section{Authors' contributions}

All authors contributed to the study conception and design. KDK, KMP, and LGK performed the literature search and data collection. All authors contributed to data analysis. The first draft of the manuscript was written by KMF, KDK, and KMP, and all authors commented on previous versions of the manuscript. All authors read and approved the final manuscript. JPL had full access to all the data in the study and takes responsibility for the integrity of the data and the accuracy of the data analysis.

\section{Funding}

This work was supported by the Canadian Institutes of Health Research Canadian 2019 Novel Coronavirus (COVID-2019) Rapid Research Funding Opportunity - Operating Grant (grant number RN420046-439965) to JPL. The funder of the study had no role in study design, data collection, data analysis, data interpretation, or writing of the report. KKSP is a member of the advisory board for Elsius Biomedical but has received no direct or indirect compensation and has received research grant funding from the $\mathrm{CIHR}$ and the MSI Foundation. All other authors declare no support from any organization for the submitted work, no financial relationships with any organizations that might have an interest in the submitted work in the previous 3 years, and no other relationships or activities that could appear to have influenced the submitted work.

\section{Availability of data and materials}

Data sharing is not applicable to this article as no datasets were generated or analyzed during the current study.
Ethics approval and consent to participate

Not applicable

\section{Consent for publication}

Not applicable

\section{Competing interests}

KKSP is a member of the advisory board for Elsius Biomedical but has received no direct or indirect compensation and has received research grant funding from the CIHR and the MSI Foundation. All other authors declare no support from any organization for the submitted work, no financial relationships with any organizations that might have an interest in the submitted work in the previous 3 years, and no other relationships or activities that could appear to have influenced the submitted work.

\section{Author details}

'Department of Critical Care Medicine, Cumming School of Medicine, University of Calgary \& Alberta Health Services, 3134 Hospital Drive NW, Calgary T2N4Z6, Canada. ${ }^{2}$ Department of Community Health Sciences and O'Brien Institute for Public Health, Cumming School of Medicine, University of Calgary, 3134 Hospital Drive NW, Calgary T2N4Z6, Canada. ${ }^{3}$ Department of Surgery, Cumming School of Medicine, University of Calgary \& Alberta Health Services, 3134 Hospital Drive NW, Calgary T2N4Z6, Canada. ${ }^{4}$ Department of Anaesthesia, Cumming School of Medicine, University of Calgary \& Alberta Health Services, 3134 Hospital Drive NW, Calgary T2N4Z6, Canada. ${ }^{5}$ Faculty of Health, School of Health Administration, Dalhousie University, 5850 College Street, Halifax, Nova Scotia B3H4R2, Canada. ${ }^{6}$ Department of Critical Care Medicine, Faculty of Medicine, Dalhousie University, 6299 South St, Halifax, Nova Scotia B3H4R2, Canada.

Received: 23 September 2020 Accepted: 25 November 2020

Published online: 18 December 2020

\section{References}

1. Kain T, Fowler R. Preparing intensive care for the next pandemic influenza. Crit Care. 2019;23(1):337

2. Center for Disease Control and Prevention NCflaRD. Past Pandemics 2018 [Available from: https://www.cdc.gov/flu/pandemic-resources/basics/pastpandemics.html]. (Accessed 16 Nov 2020).

3. Kilbourne ED. Influenza pandemics of the 20th century. Emerg Infect Dis. 2006;12(1):9-14.

4. Organization WH. Rolling updates on coronavirus disease (COVID-19) 2020 [Available from: https://www.who.int/emergencies/diseases/novelcoronavirus-2019/events-as-they-happen. (Accessed 16 Nov 2020).

5. Herold S, Becker C, Ridge KM, Budinger GR. Influenza virus-induced lung injury: pathogenesis and implications for treatment. Eur Respir J. 2015;45(5): 1463-78.

6. Marshall JC, Bosco, L., Adhikari, N. K., Connolly, B., Diaz, J. V., Dorman, T., Fowler, R. A., Meyfroidt, G., Nakagawa, S., Pelosi, P., Vincent, J. L., Vollman, K., \& Zimmerman, J. What is an intensive care unit? A report of the task force of the World Federation of Societies of Intensive and Critical Care Medicine. J Critical Care. 2017;37:270-6.

7. Ma X, Vervoort D. Critical care capacity during the COVID-19 pandemic: global availability of intensive care beds. J Crit Care. 2020;58:96-7.

8. Worldometers.info. Worldometer Dover, Delaware, U.S.A.2020 [Available from: https://www.worldometers.info/. (Accessed 16 Nov 2020).

9. News W. World is ill-prepared for "inevitable" flu pandemic. Bull World Health Organ. 2004;82(4):317-8.

10. Emanuel EJ, Persad G, Upshur R, Thome B, Parker M, Glickman A, et al. Fair allocation of scarce medical resources in the time of COVID-19. N Engl J Med. 2020;382(21):2049-55.

11. Christian MD, Sprung CL, King MA, Dichter JR, Kissoon N, Devereaux AV et al. Triage: care of the critically ill and injured during pandemics and disasters: CHEST consensus statement. Chest. 2014;146(4 Suppl):e61S-74S.

12. Maves RC, Downar J, Dichter JR, Hick JL, Devereaux A, Geiling JA, et al. Triage of scarce critical care resources in COVID-19 an implementation guide for regional allocation: an expert panel report of the Task Force for Mass Critical Care and the American College of Chest Physicians. Chest. 2020;158(1):212-25. 
13. Dahine J, Hébert PC, Ziegler D, Chenail N, Ferrari N, Hébert R. Practices in triage and transfer of critically ill patients: a qualitative systematic review of selection criteria. Crit Care Med. 2020.

14. Leider JP, DeBruin D, Reynolds N, Koch A, Seaberg J. Ethical guidance for disaster response, specifically around crisis standards of care: a systematic review. Am J Public Health. 2017;107(9):e1-9.

15. Antommaria AHM, Gibb TS, McGuire AL, Wolpe PR, Wynia MK, Applewhite MK, et al. Ventilator triage policies during the COVID-19 pandemic at U.S. hospitals associated with members of the association of bioethics program directors. Ann Intern Med. 2020;173(3):188-194.

16. Moher D, Liberati A, Tetzlaff J, Altman DG, Group P. Preferred reporting items for systematic reviews and meta-analyses: the PRISMA statement. BMJ. 2009;339:b2535.

17. Moola S MZ, Tufanaru C, Aromataris E, Sears K, Sfetcu R, Currie M, Qureshi R, Mattis P, Lisy K, Mu P-F. Chapter 7: Systematic reviews of etiology and risk. In: Aromataris E MZ, editor. Joanna Briggs Institute Reviewer's Manual: The Joanna Briggs Institute; 2017.

18. Lockwood CMZ, Porritt K. Qualitative research synthesis: methodological guidance for systematic reviewers utilizing meta-aggregation. Int J Evidence Based Healthcare. 2015;13(3):179-87.

19. McArthur AKJ, Yan H, Florescu S. Innovations in the systematic review of text and opinion. Int J Evid Evide Based Healthc. 2015;13(3):188-95.

20. Tufanaru C MZ, Aromataris E, Campbell J, Hopp L. Chapter 3: Systematic reviews of effectiveness. . In: Aromataris E MZ, editor. Joanna Briggs Institute Reviewer's Manual: The Joanna Briggs Institute; 2017.

21. Whiting PF RA, Westwood ME, Mallett S, Deeks JJ, Reitsma JB, Leeflang MM, Sterne JA, Bossuyt PM, QUADAS-2 Group. QUADAS-2: a revised tool for the quality assessment of diagnostic accuracy studies. Ann Intern Med 2011 155(8):529-536.

22. Campbell JMKM, Ding S, Carmody DP, Hakonsen SJ, Jadotte YT, White S, Munn Z. Diagnostic test accuracy: methods for systematic review and metaanalysis. Int J Evidence Based Healthcare. 2015;13(3):154-62

23. Lin JY, Anderson-Shaw L. Rationing of resources: ethical issues in disasters and epidemic situations. Prehosp Disasted Med. 2009;24(3):215-21.

24. Powell T, Christ KC, Birkhead GS. Allocation of ventilators in a public health disaster. Disaster Med Public Health Prep. 2008;2(1):20-6.

25. Prekker ME, Brunsvold ME, Bohman JK, Fischer G, Gram KL, Litell JM, et al. Regional planning for extracorporeal membrane oxygenation allocation during COVID-19. Chest. 2020.

26. Utley M, Pagel C, Peters MJ, Petros A, Lister P. Does triage to critical care during a pandemic necessarily result in more survivors? Crit Care Med. 2011; 39(1):179-83.

27. Kanter RK. Would triage predictors perform better than first-come, firstserved in pandemic ventilator allocation? Chest. 2015;147(1):102-8.

28. Christian MD, Hawryluck L, Wax RS, Cook T, Lazar NM, Herridge MS, et al. Development of a triage protocol for critical care during an influenza pandemic. CMAJ. 2006;175(11):1377-81

29. Valiani S, Terrett L, Gebhardt C, Prokopchuk-Gauk O, Isinger M Development of a framework for critical care resource allocation for the COVID-19 pandemic in Saskatchewan. CMAJ. 2020;192(37):E1067-E73.

30. Janig C, Forklage R, Gurney JM, Groth R, Wirth C, van de Krol H, et al. Triage decisions in the context of COVID-19: old burden, new challenge-the Structured Approach for Intensive Care Unit Triage (SAINT) protocol. Mil Med. 2020;02:02.

31. Ehmann MR, Zink EK, Levin AB, Suarez Jl, Belcher HM, Daugherty Biddison $E L$, et al. Operational recommendations for scarce resource allocation in a public health crisis. Chest. 2020;26:26.

32. Real de Asua D, Galvan JM, Iglesias J, Fernandez J, Maria Galvan J, Diego R, et al. Triage criteria for cardiopulmonary resuscitation and advanced life support during the COVID-19 epidemic. Med Clin (Barc). 2020;155(5):215-9.

33. Sprung CL, Joynt GM, Christian MD, Truog RD, Rello J, Nates JL. Adult ICU triage during the coronavirus disease 2019 pandemic: who will live and who will die? Recommendations to Improve Survival. Crit Care Med. 2020; 48(8):1196-1202

34. Kaposy C, Bandrauk N, Pullman D, Singleton R, Brunger F. Adapting the Hamilton Health Sciences critical care pandemic triage protocol. Healthc Q. 2010;13(2):60-3

35. Han SA, Koch VG. Clinical and Ethical Considerations in Allocation of Ventilators in an Influenza Pandemic or Other Public Health Disaster: A Comparison of the 2007 and. New York State Ventilator Allocation Guidelines. Disaster Med Public Health Prep. 2015:2020:1-27.
36. Frolic A, Kata A, Kraus P. Development of a critical care triage protocol for pandemic influenza: integrating ethics, evidence and effectiveness. Healthc Q. 2009;12(4):54-62.

37. Warrillow S, Austin D, Cheung W, Close E, Holley A, Horgan B, et al. ANZICS guiding principles for complex decision making during the COVID-19 pandemic. Crit Care Resusc. 2020;22(2):98-102.

38. Sprung CL, Zimmerman JL, Christian MD, Joynt GM, Hick JL, Taylor B, et al. Recommendations for intensive care unit and hospital preparations for an influenza epidemic or mass disaster: summary report of the European Society of Intensive Care Medicine's Task Force for intensive care unit triage during an influenza epidemic or mass disaster. Intensive Care Med. 2010; 36(3):428-43.

39. Cheung WK, Myburgh J, Seppelt IM, Parr MJ, Blackwell N, Demonte S, et al. A multicentre evaluation of two intensive care unit triage protocols for use in an influenza pandemic. Med J Aust. 2012;197(3):178-81.

40. Ashton-Cleary D, Tillyard A, Freeman N. Intensive care admission triage during a pandemic: a survey of the acceptability of triage tools. J Intensive Care Soc. 2011;12(3):180-6.

41. Marriott D, Turner Z, Robin N, Singh S. To admit or not to admit? The suitability of critical care admission criteria. Crit Care. 2012;Conference:32nd International Symposium on Intensive Care and Emergency Medicine. Brussels Belgium. Conference Publication: (var.pagings). 16 (SUPPL. 1) (pp S181-S2).

42. Leclerc T, Donat N, Donat A, Pasquier P, Libert N, Schaeffer E, et al. Prioritisation of ICU treatments for critically ill patients in a COVID-19 pandemic with scarce resources. Anaesth Crit Care Pain Med. 2020;39(3): 333-9.

43. Hick JL, O'Laughlin DT. Concept of operations for triage of mechanical ventilation in an epidemic. Acad Emerg Med. 2006;13(2):223-9.

44. Azoulay É, Beloucif S, Guidet B, Pateron D, Vivien B, Le Dorze M. Admission decisions to intensive care units in the context of the major COVID-19 outbreak: local guidance from the COVID-19 Paris-region area. Crit Care. 2020;24(1):293.

45. Herreros B, Gella P, Real de Asua D. Triage during the COVID-19 epidemic in Spain: better and worse ethical arguments. J Med Ethics 2020;46(7):455-458.

46. Marckmann G, Neitzke G, Schildmann J, Michalsen A, Dutzmann J, Hartog C, et al. Decisions on the allocation of intensive care resources in the context of the COVID-19 pandemic : clinical and ethical recommendations of DIVI, DGINA, DGAI, DGIIN, DGNI, DGP, DGP and AEM. Med Klin Intensivmed Notfmed 2020:29:29.

47. Committee PCPE. Interim ethical recommendations in medical management in the COVID-19 crisis. Philipp J Intern Med. 2020;58(1):VIII-XIV .

48. Cheung W, Myburgh J, Seppelt IM, Parr MJ, Blackwell N, Demonte S, et al. Development and evaluation of an influenza pandemic intensive care unit triage protocol. Crit Care Resusc. 2012;14(3):185-90.

49. Swiss Academy of Medical Sciences. COVID-19 pandemic: triage for intensive-care treatment under resource scarcity. Swiss Med Wkly. 2020;150: w20229.

50. Vincent JL, Creteur J. Ethical aspects of the COVID-19 crisis: how to deal with an overwhelming shortage of acute beds. Eur Heart J Acute Cardiovasc Care. 2020:9(3):248-52.

51. Gupta S, Batt J, Bourbeau J, Chapman KR, Gershon A, Granton J, et al. Triaging access to critical care resources in patients with chronic respiratory diseases in the event of a major COVID-19 surge: key highlights from the Canadian Thoracic Society (CTS) Position Statement. Chest. 2020;18:18.

52. Montgomery J, Stokes-Lampard HJ, Griffiths MD, Gardiner D, Harvey D, Suntharalingam G. Assessing whether COVID-19 patients will benefit from critical care, and an objective approach to capacity challenges during a pandemic: an Intensive Care Society clinical guideline. J Intensive Care Soc. 2020; https://doi.org/10.1177/1751143720948537.

53. Vergano M, Bertolini G, Giannini A, Gristina GR, Livigni S, Mistraletti G, et al. SIAARTI recommendations for the allocation of intensive care treatments in exceptional, resource-limited circumstances. Minerva Anestesiol. 2020;86(5): 469-72.

54. Murad H, Kaufman D, Greenlaw J, Harvey T, D'Angio C, Demme R. Assessment of the new york state department of health proposed guidelines for ventilator allocation in the scenario of severe influenza pandemic. Crit Care Med. 2009;Conference:39th Critical Care Congress of the Society of Critical Care Medicine's. Miami Beach, FL United States. Conference Publication: (var.pagings). 7 (12 SUPPL.) (pp A387). 
55. Rowan KM, Harrison DA, Walsh TS, McAuley DF, Perkins GD, Taylor BL, et al. The Swine Flu Triage (SwiFT) study: development and ongoing refinement of a triage tool to provide regular information to guide immediate policy and practice for the use of critical care services during the H1N1 swine influenza pandemic. Health Technol Assess Rep. 2011;14(55):335-492.

56. Wilkens EP, Klein GM. Mechanical ventilation in disaster situations: a new paradigm using the AGILITIES Score System. Am J Disaster Med. 2010;5(6): 369-84.

57. Myles PR, Nguyen-Van-Tam JS, Lim WS, Nicholson KG, Brett SJ, Enstone JE, et al. Comparison of CATs, CURB-65 and PMEWS as triage tools in pandemic influenza admissions to UK hospitals: case control analysis using retrospective data. PLoS ONE. 2012;7(4):e34428.

58. Daugherty Biddison EL, Faden R, Gwon HS, Mareiniss DP, Regenberg AC, Schoch-Spana M, et al. Too many patients...a framework to guide statewide allocation of scarce mechanical ventilation during disasters. Chest. 2019; 155(4):848-854.

59. Challen K, Bright J, Bentley A, Walter D. Physiological-social score (PMEWS) vs. CURB-65 to triage pandemic influenza: a comparative validation study using community-acquired pneumonia as a proxy. BMC Health Serv Res. 2007;7(no pagination).

60. Muller MP, McGeer AJ, Hassan K, Marshall J, Christian M, Toronto Invasive Bacterial Disease N. Evaluation of pneumonia severity and acute physiology scores to predict ICU admission and mortality in patients hospitalized for influenza. PLoS ONE. 2010;5(3):e9563.

61. Adeniji KA, Cusack R. The Simple Triage Scoring System (STSS) successfully predicts mortality and critical care resource utilization in H1N1 pandemic flu: a retrospective analysis. Crit Care. 2011;15(1):R39.

62. Talmor D, Jones AE, Rubinson L, Howell MD, Shapiro NI. Simple triage scoring system predicting death and the need for critical care resources for use during epidemics. Crit Care Med. 2007;35(5):1251-6.

63. Morton B, Tang L, Gale R, Kelly M, Robertson H, Mogk M, et al. Performance of influenza-specific triage tools in an H1N1-positive cohort: P/F ratio better predicts the need for mechanical ventilation and critical care admission. $\mathrm{Br} J$ Anaesth. 2015;114(6):927-33.

64. Morton B, Tang L, Gale R, Kelly M, Robertson H, Williams K, et al. Do pandemic influenza specific triaging tools perform better than conventional severity scores? A retrospective cohort study. Intensive Care Med. 2014; Conference:27th Annual Congress of the European Society of Intensive Care Medicine, ESICM 2014. Barcelona Spain. Conference Publication: (var. pagings). 40 (1 SUPPL. 1) (pp S65).

65. Ibrahim H, Sorrell S, Nair SC, Al Romaithi A, Al Mazrouei S, Kamour A. Rapid development and utilization of a clinical intelligence dashboard for frontline clinicians to optimize critical resources during COVID-19. Acta Informatica Medica. 2020;28(3):209-13.

66. Ryan L, Lam C, Mataraso S, Allen A, Green-Saxena A, Pellegrini E, et al. Mortality prediction model for the triage of COVID-19, pneumonia, and mechanically ventilated ICU patients: a retrospective study. Ann Med. 2020; 59:207-16.

67. Commons RJ, Denholm J. Triaging pandemic flu: pneumonia severity scores are not the answer. Int J Tuberc Lung Dis. 2012;16(5):670-3.

68. Estella A. Usefulness of CURB-65 and pneumonia severity index for influenza a H1N1v pneumonia. Monaldi Arch Chest Dis. 2012;77(3-4):118-21.

69. Pereira JM, Moreno RP, Matos R, Rhodes A, Martin-Loeches I, Cecconi M, et al. Severity assessment tools in ICU patients with 2009 influenza A (H1N1) pneumonia. Clin Microbiol Infex. 2012;18(10):1040-8.

70. Enfield K, Miller R, Rice T, Thompson BT, Truwit J. Limited utility of SOFA and APACHE $\|$ prediction models for ICU triage in pandemic influenza. Chest. 2011;Conference:CHEST 2011. Honolulu, HI United States. Conference Publication: (var.pagings). 140 (4 MEETING ABSTRACT) (no pagination).

71. Saleh A, Ahmed M, Abdel-Lateif A. Comparison of the mortality prediction of different ICU scoring systems (apache II and III, SAPS II, and sofa) in acute respiratory distress syndrome patients. Chest. 2016;Conference:CHEST World Congress 2016 Annual Meeting. Shanghai China. Conference Publication: (var.pagings). 149 (4 SUPPL. 1) A147.

72. Grissom CK, Brown SM, Kuttler KG, Boltax JP, Jones J, Jephson AR, et al. A modified Sequential Organ Failure Assessment score for critical care triage. Disaster Med Public Health Prep. 2010;4(4):277-84.

73. Khan Z, Hulme J, Sherwood N. An assessment of the validity of SOFA score based triage in H1N1 critically ill patients during an influenza pandemic. Anaesthesia. 2009;64(12):1283-8.
74. Miller RR, Brown S, Enfield KB, Truwit JD, Dascomb KK, Jephson AR, et al. Overestimation of mortality by a SOFA-based influenza triage tool compared to intensive care unit mortality experienced during the early 2009 novel H1N1 influenza epidemic in Utah. Am J Respir Crit Care Med. 2010;Conference:American Thoracic Society International Conference, ATS 2010. New Orleans, LA United States. Conference Publication: (var.pagings). 181 (1 MeetingAbstracts) (no pagination).

75. Shahpori R, Stelfox HT, Doig CJ, Boiteau PJE, Zygun DA. Sequential Organ Failure Assessment in H1N1 pandemic planning. Crit Care Med. 2011;39(4): 827-32.

76. Williams L, Gannon J. Use of the SOFA score in pandemic influenza - a prospective study. J Intensive Care Soc. 2009;10(3):179-82.

77. Adalja AA, Watson M, Waldhorn RE, Toner ES. A conceptual approach to improving care in pandemics and beyond: severe lung injury centers. J Crit Care. 2013;28(3):318.e9-15.

78. Guest T, Tantam G, Donlin N, Tantam K, McMillan H, Tillyard A. An observational cohort study of triage for critical care provision during pandemic influenza: 'clipboard physicians' or 'evidenced based medicine'? Anaesthesia. 2009;64(11):1199-206.

79. Christian MD, Hamielec C, Lazar NM, Wax RS, Griffith L, Herridge MS, et al. A retrospective cohort pilot study to evaluate a triage tool for use in a pandemic. Crit Care. 2009;13(5):R170.

80. O'Laughlin DT, Hick JL. Ethical issues in resource triage. Respir Care. 2008; 53(2):190-7 discussion 7-200.

81. Farrell TW, Francis $L$, Brown T, Ferrante LE, Widera E, Rhodes R, et al. Rationing limited healthcare resources in the COVID-19 era and beyond: ethical considerations regarding older adults. J Am Geriatr Soc. 2020;68(6): 1143-9.

82. Seethala R, Keller SP. Extracorporeal membrane oxygenation resource planning in the setting of pandemic respiratory illness. Ann Am Thorac Soc. 2020;17(7):800-3.

83. Rhodes KV, Wei EK, Salway RJ, Natsui S, Silvestri D, Cassel CK. The New York City pandemic resuscitation equitable allocation principles. Resuscitation. 2020;156:123-4.

84. Steinberg A, Levy-Lahad E, Karni T, Sprung CL. Israeli position paper: triage decisions for severely ill patients during the COVID-19 pandemic. Chest. 2020;158(6):2278-81.

85. Christian MD, Joynt GM, Hick JL, Colvin J, Danis M, Sprung CL, et al. Chapter 7. Critical care triage. Recommendations and standard operating procedures for intensive care unit and hospital preparations for an influenza epidemic or mass disaster. Intensive Care Med. 2010;36 Suppl 1:S55-64.

86. Cinti SK, Barnosky AR, Gay SE, Goold SD, Lozon MM, Kim K, et al. Bacterial pneumonias during an influenza pandemic: how will we allocate antibiotics? Biosecur Bioterror. 2009:7(3):311-6.

87. Daugherty Biddison EL, Gwon H, Schoch-Spana M, Cavalier R, White DB, Dawson T, et al. The community speaks: understanding ethical values in allocation of scarce lifesaving resources during disasters. Ann Am Thorac Soc. 2014;11(5):777-83.

88. Daugherty Biddison EL, Berkowitz KA, Courtney B, De Jong MJ, Devereaux $A V$, Kissoon N, et al. Ethical considerations: care of the critically ill and injured during pandemics and disasters: CHEST consensus statement. Chest. 2014;146(Supplement 4):e145S-55S.

89. Devereaux AV, Dichter JR, Christian MD, Dubler NN, Sandrock CE, Hick JL, et al. Definitive care for the critically III during a disaster: a framework for allocation of scarce resources in mass critical care. Chest. 2008;133(5 SUPPL): 51S-66S.

90. Eastman N, Philips B, Rhodes A. Triaging for adult critical care in the event of overwhelming need. Intensive Care Med. 2010;36(6):1076-82.

91. Einav S, Hick JL, Hanfling D, Erstad BL, Toner ES, Branson RD, et al. Surge capacity logistics: care of the critically ill and injured during pandemics and disasters: CHEST consensus statement. Chest. 2014;146(4 Suppl):e17S-43S.

92. Rubinson L, Nuzzo JB, Talmor DS, O'Toole T, Kramer BR, Inglesby TV. Augmentation of hospital critical care capacity after bioterrorist attacks or epidemics: recommendations of the Working Group on Emergency Mass Critical Care. Crit Care Med. 2005;33(10):2393-403.

93. Silva DS, Gibson JL, Robertson A, Bensimon CM, Sahni S, Maunula L, et al. Priority setting of ICU resources in an influenza pandemic: a qualitative study of the Canadian public's perspectives. BMC Public Health. 2012:12:241.

94. Cheung W, Myburgh J, McGuinness S, Chalmers D, Parke R, Blyth F, et al. A cross-sectional survey of Australian and New Zealand public opinion on 
methods to triage intensive care patients in an influenza pandemic. Critical Care Resuscitation. 2017;19(3):254-65.

95. Cheung W, Myburgh J, McGuinness S, Chalmers D, Parke R, Blyth F, et al. A cross-sectional survey of Australian and New Zealand public opinion on methods to triage intensive care patients in an influenza pandemic. Crit Care Resusc. 2017:19(3):254-65.

96. Dries D, Reed MJ, Kissoon N, Christian MD, Dichter JR, Devereaux AV, et al. Special populations: care of the critically ill and injured during pandemics and disasters: CHEST consensus statement. Chest. 2014;146(4 Suppl):e75S865 .

97. Tillyard A. Reorganising the pandemic triage processes to ethically maximise individuals' best interests. Intensive Care Med. 2010;36(11):1966-71.

98. Daugherty Biddison ELD, Gwon HS, Schoch-Spana M, Regenberg AC, Juliano C, Faden RR, et al. Scarce resource allocation during disasters: a mixed-method community engagement study. Chest. 2018;153(1):187-95.

99. Tabery J, Mackett ICW. Ethics of triage in the event of an influenza pandemic. Disaster Med Public Health Prep. 2008;2(2):114-8.

100. Christian MD, Devereaux AV, Dichter JR, Rubinson L, Kissoon N, Task Force for Mass Critical C, et al. Introduction and executive summary: care of the critically ill and injured during pandemics and disasters: CHEST consensus statement. Chest. 2014;146(4 Suppl):8S-34S.

101. Ardagh M. Criteria for prioritising access to healthcare resources in New Zealand during an influenza pandemic or at other times of overwhelming demand. N Z Med J. 2006;119(1243):U2256.

102. Shekar K, Badulak J, Peek G, Boeken U, Dalton HJ, Arora L, et al. Extracorporeal life support organization coronavirus disease 2019 interim guidelines: a consensus document from an international group of interdisciplinary extracorporeal membrane oxygenation providers. ASAIO J 2020;66(7):707-21.

103. Winsor S, Bensimon CM, Sibbald R, Anstey K, Chidwick P, Coughlin K, et al. Identifying prioritization criteria to supplement critical care triage protocols for the allocation of ventilators during a pandemic influenza. Healthc Q. 2014;17(2):44-51.

104. Angus DC, Marrie TJ, Obrosky DS, Clermont G, Dremsizov TT, Coley C, et al. Severe community-acquired pneumonia: use of intensive care services and evaluation of American and British Thoracic Society Diagnostic criteria. Am J Respir Crit Care Med. 2002;166(5):717-23.

105. Jones AE, Trzeciak S, Kline JA. The Sequential Organ Failure Assessment score for predicting outcome in patients with severe sepsis and evidence of hypoperfusion at the time of emergency department presentation. Crit Care Med. 2009;37(5):1649-54

106. Alvarez M, Nava JM, Rué M, Quintana S. Mortality prediction in head trauma patients: performance of Glasgow Coma Score and general severity systems. Crit Care Med. 1998;26(1):142-8.

107. Tambone V, Boudreau D, Ciccozzi M, Sanders K, Campanozzi LL, Wathuta J, et al. Ethical Criteria for the Admission and Management of Patients in the ICU Under Conditions of Limited Medical Resources: A Shared International Proposal in View of the COVID-19 Pandemic. Front Public Health. 2020;8: 284.

108. Schuetz P, Koller M, Christ-Crain M, Steyerberg E, Stolz D, Müller C, et al. Predicting mortality with pneumonia severity scores: importance of model recalibration to local settings. Epidemiol Infect. 2008;136(12):1628-37.

109. Wynants L, Van Calster B, Bonten MMJ, Collins GS, Debray TPA, De Vos M, et al. Prediction models for diagnosis and prognosis of COVID-19 infection: systematic review and critical appraisal. BMJ. 2020;369:m1328.

110. Ornelas J, Dichter JR, Devereaux AV, Kissoon N, Livinski A, Christian MD, et al. Methodology: care of the critically ill and injured during pandemics and disasters: CHEST consensus statement. Chest. 2014;146(4 Suppl):35S$41 \mathrm{~S}$.

111. Ahmed I. COVID-19 - does exercise prescription and maximal oxygen uptake (VO2 max) have a role in risk-stratifying patients? J R Coll Physicians Lond. 2020;20(3):282-4.

112. Baumrucker SJ, Carter G, Adkins RW, Perkins C, Stolick M, VandeKieft G. Ethics roundtable: distribution of critical care resources in the setting of a COVID-19 surge. Am J Hosp Palliat Care. 2020;37(12):1096-101.

113. Challen K, Goodacre SW, Wilson R, Bentley A, Campbell M, Fitzsimmons C, et al. Evaluation of triage methods used to select patients with suspected pandemic influenza for hospital admission. EMJ. 2011;17.

114. Darvall JN, Bellomo R, Bailey M, Paul E, Young PJ, Rockwood K, et al. Frailty and outcomes from pneumonia in critical illness: a population-based cohort study. Br J Anaesth. 2020;125(5):730-8.
115. García Clemente MM, Herrero Huertas J, Fernández Fernández A, De La Escosura Muñoz C, Enríquez Rodríquez Al, Pérez Martínez L, et al. Assessment of risk scores in COVID-19. Int J Clin Pract. 2020; https://doi.org/ 10.1111/ijcp.13705.

116. Mejia-Vilet JM, Cordova-Sanchez BM, Fernandez-Camargo DA, Mendez-Perez RA, Morales-Buenrostro LE, Hernandez-Gilsoul T. A risk score to predict admission to the intensive care unit in patients with COVID-19: the ABCGOALS score. Salud Publ Mex. 2020;10:10.

117. Nicholson CJ, Wooster L, Sigurslid HH, Li RF, Jiang W, Tian W, et al. Estimating risk of mechanical ventilation and mortality among adult COVID19 patients admitted to Mass General Brigham: the VICE and DICE scores. Medrxiv. 2020;16:16.

118. Walsh B, Mukherjee V, Uppal A. An observational cohort study: how would resource allocation policies perform during a surge of patients with COVID19? Chest. 2020;158(4 Supplement):A1173.

119. Wu G, Yang P, Xie Y, Woodruff HC, Rao X, Guiot J, et al. Development of a clinical decision support system for severity risk prediction and triage of COVID-19 patients at hospital admission: an international multicenter study. Eur Respir J. 2020;02:02.

120. Moodley K, Ravez L, Obasa AE, Mwinga A, Jaoko W, Makindu D, et al. What could "fair allocation" during the COVID-19 crisis possibly mean in subSaharan Africa? Hast Cent Rep. 2020:50(3):33-5.

121. Tricco AC, Antony J, Zarin W, Strifler L, Ghassemi M, Ivory J, et al. A scoping review of rapid review methods. BMC Med. 2015;13:224.

122. Killien EY, Mills B, Errett NA, Sakata V, Vavilala MS, Rivara FP, Kissoon N, King MA. Prediction of pediatric critical care resource utilization for disaster triage. Pediatric Critical Care Med. 2020;21(8):e491-501.

123. Kim KM, Cinti S, Gay S, Goold S, Barnosky A, Lozon M. Triage of mechanical ventilation for pediatric patients during a pandemic. Disaster Med Public Health Preparedness. 2012;6(2):131-1378.

124. Barie PS, Bronstein M, Gibson CJ, Kelly AG, Lee C, Narayan M, Shou J, Smith KE, Villegas C, Winchell RJ. A Simple Three-Tier Classification System for Triage, Communication, and Resource Utilization by Patients Afflicted with COVID-19 Disease. Surg Infect (Larchmt). 2020;21 (8):726-7.

125. Brandão-Neto RA, Goulart AC, Santana AN, Martins HS, Ribeiro SC, Ho LY, Chiamolera M, Magri MM, Scalabrini-Neto A, Velasco IT. The role of pneumonia scores in the emergency room in patients infected by 2009 H1N1 infection. Eur J Emerg Med. 2012;19(3):200-2.

\section{Publisher's Note}

Springer Nature remains neutral with regard to jurisdictional claims in published maps and institutional affiliations.
Ready to submit your research? Choose BMC and benefit from:

- fast, convenient online submission

- thorough peer review by experienced researchers in your field

- rapid publication on acceptance

- support for research data, including large and complex data types

- gold Open Access which fosters wider collaboration and increased citations

- maximum visibility for your research: over $100 \mathrm{M}$ website views per year

At BMC, research is always in progress.

Learn more biomedcentral.com/submissions 